\title{
Copper-Catalyzed Denitrogenative Transannulation Reaction of Pyridotriazoles: Synthesis of Imidazopyridines with Amines and Amino acids
}

Abhisek Joshi, Darapaneni Chandra Mohan and Subbarayappa Adimurthy*

Academy of Scientific \& Innovative Research, CSIR-Central Salt \& Marine Chemicals Research Institute, G. B. Marg, Bhavnagar-364 002. Gujarat (INDIA)

E-mail: adimurthy@csmcri.org

\section{Table of Contents}

Experimental Section

$\mathrm{S} 2$

General procedure

$\mathrm{S} 2$

Characterization data

S3-S15

${ }^{1} \mathrm{H} \&{ }^{13} \mathrm{C}$ - NMR Spectra of all products

S16-S40 


\section{Experimental Section}

General: All commercially available chemicals and reagents were used without any further purification unless otherwise indicated. ${ }^{1} \mathrm{H}$ and ${ }^{13} \mathrm{C}$ NMR spectra were recorded at 500 and 125 $\mathrm{MHz}$ respectively. The spectra were recorded in $\mathrm{CDCl}_{3}$ and $\mathrm{DMSO}-\mathrm{d}_{6}$ as solvent. Multiplicity was indicated as follows: $\mathrm{s}$ (singlet); $\mathrm{d}$ (doublet); $\mathrm{t}$ (triplet); $\mathrm{m}$ (multiplet); dd (doublet of doublets), etc. and coupling constants ( $\mathbf{J})$ were given in Hz. Chemical shifts are reported in ppm relative to TMS as an internal standard. The peaks around delta values of ${ }^{1} \mathrm{H}$ NMR (7.2), and ${ }^{13} \mathrm{C}$ NMR (77.0) are correspond to deuterated solvent chloroform 1H NMR (2.50) and 13C NMR (39.43) are correspond to deuterated solvent DMSO respectively. Mass spectra were obtained using electron impact (EI) ionization method. Progress of the reactions was monitored by thin layer chromatography (TLC). All products were purified through column chromatography using silica gel 100-200 mesh size using hexane/ethyl acetate as eluent unless otherwise indicated.

\section{General procedure}

General procedure for the synthesis of imidazo[1,5-a]pyridine (3a): To a reaction tube equipped with a magnetic stir bar, added 3-phenyl-[1,2,3]triazolo[1,5-a]pyridine 1a $(39.0 \mathrm{mg}$, $0.2 \mathrm{mmol})$, benzyl amine $2 \mathbf{a}(64.2 \mathrm{mg}, 0.6 \mathrm{mmol})$, and copper iodide $(0.04 \mathrm{mmol} / 20 \mathrm{~mol} \%)$ and $1.0 \mathrm{~mL}$ of 1,2 -dichlorobenzne. The mixture was heated in an oil bath at $150{ }^{\circ} \mathrm{C}$ in a closed tube. Reaction was monitored by TLC, after completion of the reaction, it was allowed to attain room temperature. Then the mixture was poured into $30 \mathrm{~mL}$ of sodium chloride solution. The product was extracted with EtOAc $\left(15 \mathrm{~mL} \mathrm{X} \mathrm{3)}\right.$ and dried with anhydrous $\mathrm{Na}_{2} \mathrm{SO}_{4}$. Removal of the solvent under reduced pressure the left out residue was purified by column chromatography using silica gel (4\% EtOAc/hexane) to afford 3a (44.5 mg; 82\% yield). 


\section{Characterization data}

\section{1, 3-diphenylimidazo[1,5-a]pyridine (3a)}

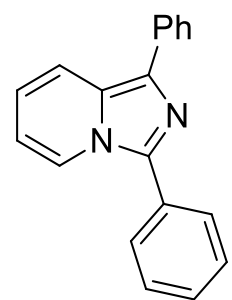

(Eluent: 4\% EtOAc/hexane); $82 \%$ yield (44.5 mg); solid; ${ }^{1} \mathrm{H}$ NMR (500 MHz, $\left.\mathrm{CDCl}_{3}\right) \delta 8.21$ $(\mathrm{d}, J=5.5 \mathrm{~Hz}, 1 \mathrm{H}), 7.94(\mathrm{~d}, J=5.5 \mathrm{~Hz}, 2 \mathrm{H}), 7.81(\mathrm{~s}, 5 \mathrm{H}), 7.51(\mathrm{~d}, J=5.5 \mathrm{~Hz}, 2 \mathrm{H}), 7.45(\mathrm{~d}, J$ $=5.5 \mathrm{~Hz}, 2 \mathrm{H}), 7.29(\mathrm{~s}, 1 \mathrm{H}), 6.75(\mathrm{~s}, 1 \mathrm{H}), 6.53(\mathrm{~s}, 1 \mathrm{H}) .{ }^{13} \mathrm{C} \mathrm{NMR}\left(125 \mathrm{MHz}, \mathrm{CDCl}_{3}\right) \delta 138.0$, $134.9,131.9,130.1,128.9,128.7,128.6,128.2,127.6,126.7,126.4,121.6,119.6,119.0,113.1$. HRMS calcd for $\mathrm{C}_{19} \mathrm{H}_{15} \mathrm{~N}_{2} \mathrm{Cl}: 271.1235$. Found: 271.1240 .

\section{1-Phenyl-3-(p-tolyl)imidazo[1,5-a]pyridine (3b)}

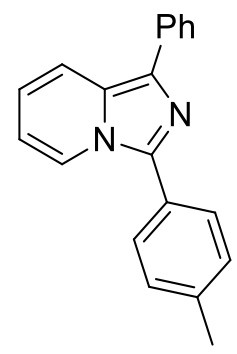

(Eluent: 4\% EtOAc/hexane); $78 \%$ yield (44.3 mg); semi-solid; ${ }^{1} \mathrm{H}$ NMR (500 MHz, $\mathrm{CDCl}_{3}$ ) $\delta 8.19(\mathrm{~d}, J=7.5 \mathrm{~Hz}, 1 \mathrm{H}), 7.93(\mathrm{~d}, J=8.0 \mathrm{~Hz}, 2 \mathrm{H}), 7.82(\mathrm{~d}, J=9.5 \mathrm{~Hz}, 1 \mathrm{H}), 7.72(\mathrm{~d}, J=8.0$ $\mathrm{Hz}, 2 \mathrm{H}), 7.47$ (t, $J=7.5 \mathrm{~Hz}, 2 \mathrm{H}), 7.33(\mathrm{~d}, J=8.0 \mathrm{~Hz}, 2 \mathrm{H}), 7.30(\mathrm{t}, J=7.5 \mathrm{~Hz}, 1 \mathrm{H}), 6.76-6.73$ $(\mathrm{m}, 1 \mathrm{H}), 6.54(\mathrm{t}, J=7.0 \mathrm{~Hz}, 1 \mathrm{H}), 2.42(\mathrm{~s}, 3 \mathrm{H}) .{ }^{13} \mathrm{C} \mathrm{NMR}\left(125 \mathrm{MHz}, \mathrm{CDCl}_{3}\right) \delta 139.6,138.8$, $138.3,135.0,131.8,129.7,128.7,128.2,127.5,127.2,126.9,126.4,121.8,119.5,119.1,113.0$ 21.4. HRMS calcd for $\mathrm{C}_{20} \mathrm{H}_{17} \mathrm{~N}_{2} \mathrm{Cl}$ : 285.1392. Found: 285.1396.

1-Phenyl-3-(m-tolyl)imidazo[1,5-a]pyridine (3c) 


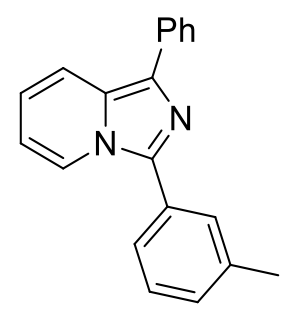

(Eluent: 4\% EtOAc/hexane); $62 \%$ yield $(35.3 \mathrm{mg})$; semi-solid; ${ }^{1} \mathrm{H}$ NMR (500 MHz, $\left.\mathrm{CDCl}_{3}\right)$ $\delta 8.19(\mathrm{~d}, J=7.0 \mathrm{~Hz}, 1 \mathrm{H}), 7.93(\mathrm{~d}, J=7.0 \mathrm{~Hz}, 2 \mathrm{H}), 7.82(\mathrm{~d}, J=9.0 \mathrm{~Hz}, 1 \mathrm{H}), 7.72(\mathrm{~d}, J=8.0$ $\mathrm{Hz}, 2 \mathrm{H}), 7.47(\mathrm{t}, J=7.5 \mathrm{~Hz}, 2 \mathrm{H}), 7.33(\mathrm{~d}, J=8.0 \mathrm{~Hz}, 2 \mathrm{H}), 7.30(\mathrm{t}, J=7.5 \mathrm{~Hz}, 1 \mathrm{H}), 6.76-6.73(\mathrm{~m}$, 1H), $6.54(\mathrm{t}, J=7.0 \mathrm{~Hz}, 1 \mathrm{H}), 2.42(\mathrm{~s}, 3 \mathrm{H}) .{ }^{13} \mathrm{C} \mathrm{NMR}\left(125 \mathrm{MHz}, \mathrm{CDCl}_{3}\right) \delta 138.8,138.3,135.0$, 131.8, 129.6, 128.7, 128.2, 127.5, 127.3, 126.8, 126.4, 121.8, 119.5, 119.1, 113.0, 21.4. HRMS calcd for $\mathrm{C}_{20} \mathrm{H}_{17} \mathrm{~N}_{2} \mathrm{Cl}$ : 285.1392. Found: 285.1386.

\section{1-Phenyl-3-(o-tolyl)imidazo[1,5-a]pyridine (3d)}

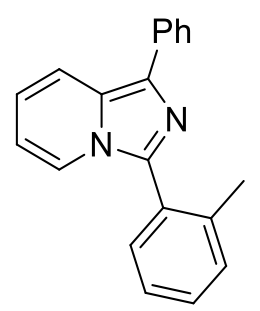

(Eluent: 4\% EtOAc/hexane); $92 \%$ yield (52.3 mg); semi-solid; ${ }^{1} \mathrm{H}$ NMR (500 MHz, $\mathrm{CDCl}_{3}$ ) $\delta 7.95(\mathrm{~d}, J=7.0 \mathrm{~Hz}, 2 \mathrm{H}), 7.85(\mathrm{~d}, J=9.0 \mathrm{~Hz}, 1 \mathrm{H}), 7.59$ (d, $J=6.5 \mathrm{~Hz}, 1 \mathrm{H}), 7.47-7.43(\mathrm{~m}$, $3 \mathrm{H}), 7.38(\mathrm{~d} J=10.0 \mathrm{~Hz}, 2 \mathrm{H}), 7.32-7.27(\mathrm{~m}, 2 \mathrm{H}), 6.78(\mathrm{t}, J=7.0 \mathrm{~Hz}, 1 \mathrm{H}), 6.51(\mathrm{t}, J=5.5 \mathrm{~Hz}$, 1H), 2.24(s, 3H). ${ }^{13} \mathrm{C}$ NMR (125 MHz, $\left.\mathrm{CDCl}_{3}\right) \delta 138.5,137.8,135.1,131.1,130.8,130.6$, 129.6, 129.2, 128.7, 126.6, 126.4, 126.1, 121.9, 119.6, 118.9, 112.9, 19.7. HRMS calcd for $\mathrm{C}_{20}$ $\mathrm{H}_{17} \mathrm{~N}_{2} \mathrm{Cl}:$ 285.1392. Found: 285.1386.

\section{3-(4-(Tert-butyl)phenyl)-1-phenylimidazo[1,5-a]pyridine (3e)}




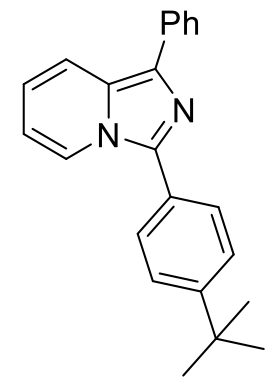

(Eluent: 4\% EtOAc/hexane); $68 \%$ yield (44.0 mg); semi-solid; ${ }^{1} \mathrm{H}$ NMR (500 MHz, $\mathrm{CDCl}_{3}$ ) $\delta 8.13(\mathrm{~d}, J=7.0 \mathrm{~Hz}, 1 \mathrm{H}), 7.84(\mathrm{~d}, J=7.5 \mathrm{~Hz}, 2 \mathrm{H}), 7.72(\mathrm{~d}, J=9.0 \mathrm{~Hz}, 1 \mathrm{H}), 7.66(\mathrm{~d}, J=8.0$ $\mathrm{Hz}, 2 \mathrm{H}), 7.45(\mathrm{~d}, J=8.0 \mathrm{~Hz}, 2 \mathrm{H}), 7.37(\mathrm{t}, J=8.0 \mathrm{~Hz}, 2 \mathrm{H}), 7.20(\mathrm{t}, J=7.0 \mathrm{~Hz}, 1 \mathrm{H}), 6.66-6.63$ $(\mathrm{m}, 1 \mathrm{H}), 6.44(\mathrm{t}, J=6.5 \mathrm{~Hz}, 1 \mathrm{H}), 1.28(\mathrm{~s}, 9 \mathrm{H}) .{ }^{13} \mathrm{C} \mathrm{NMR}\left(125 \mathrm{MHz}, \mathrm{CDCl}_{3}\right) \delta 138.2,135.0$, 131.8, 128.7, 127.2, 126.8, 126.4, 125.9, 122.9, 119.5, 119.1, 113.0, 34.8. 31.2. HRMS calcd for $\mathrm{C}_{23} \mathrm{H}_{22} \mathrm{~N}_{2} \mathrm{Cl}$ : 327.1861. Found: 327.1872 .

\section{3-(4-Methoxyphenyl)-1-phenylimidazo[1,5-a]pyridine (3f)}

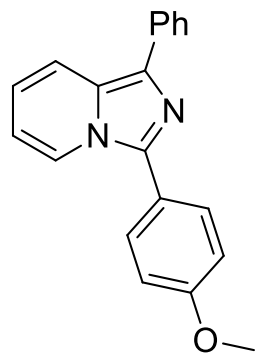

(Eluent: 4\% EtOAc/hexane); $83 \%$ yield (49.8 mg); semi-solid; ${ }^{1} \mathrm{H}$ NMR (500 MHz, $\left.\mathrm{CDCl}_{3}\right)$ $\delta 7.96(\mathrm{~d}, J=7.0 \mathrm{~Hz}, 2 \mathrm{H}), 7.86(\mathrm{~d}, J=9.0 \mathrm{~Hz}, 1 \mathrm{H}), 7.68(\mathrm{~d}, J=7.5 \mathrm{~Hz}, 1 \mathrm{H}), 7.59(\mathrm{~d}, J=6.5$ $\mathrm{Hz}, 1 \mathrm{H}), 7.49-7.43(\mathrm{~m}, 3 \mathrm{H}), 7.28(\mathrm{~m}, 1 \mathrm{H}), 7.14(\mathrm{t}, J=7.5 \mathrm{~Hz}, 1 \mathrm{H}), 7.05(\mathrm{~d}, J=8.5 \mathrm{~Hz}, 1 \mathrm{H})$, 6.81-6.78 (m, 1H), $6.54(\mathrm{t}, J=7.0 \mathrm{~Hz}, 1 \mathrm{H}), 3.81(\mathrm{~s}, 3 \mathrm{H}) .{ }^{13} \mathrm{C} \mathrm{NMR}\left(125 \mathrm{MHz}, \mathrm{CDCl}_{3}\right) \delta 157.3$, $136.0,134.9,132.8,131.2,130.9,128.6,127.3,126.7,126.3,123.5,121.2,119.5,118.8,188.5$, 112.1, 111.1, 55.5. HRMS calcd for $\mathrm{C}_{20} \mathrm{H}_{17} \mathrm{~N}_{2} \mathrm{O}: 301.1341$. Found: 301.1341 .

3-(3-Methoxyphenyl)-1-phenylimidazo[1,5-a]pyridine (3g) 


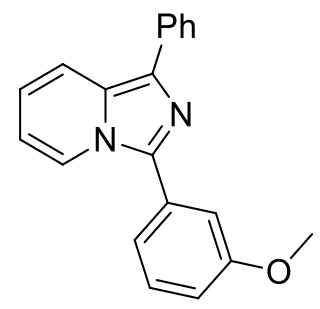

(Eluent: 4\% EtOAc/hexane); $65 \%$ yield $(39.0 \mathrm{mg})$; semi-solid; ${ }^{1} \mathrm{H} \mathrm{NMR}\left(500 \mathrm{MHz}, \mathrm{CDCl}_{3}\right)$ $\delta 8.14(\mathrm{~d}, J=7.0 \mathrm{~Hz}, 1 \mathrm{H}), 7.84(\mathrm{~d}, J=7.5 \mathrm{~Hz}, 2 \mathrm{H}), 7.72(\mathrm{~d}, J=9.5 \mathrm{~Hz}, 1 \mathrm{H}), 7.37-7.28(\mathrm{~m}$, $5 \mathrm{H}), 7.19(\mathrm{t}, J=7.0 \mathrm{~Hz}, 1 \mathrm{H}), 6.89(\mathrm{~d}, J=7.5 \mathrm{~Hz}, 1 \mathrm{H}), 6,66-6.63(\mathrm{~m}, 1 \mathrm{H}), 6.43(\mathrm{t}, J=6.5 \mathrm{~Hz}$, 1H), 3,37 (s, 3H). ${ }^{13} \mathrm{C}$ NMR (125 MHz, $\left.\mathrm{CDCl}_{3}\right) \delta 160.0,137.8,134.8,131.8,131.2,129.9$, 128.6, 127.6, 126.7, 126.4, 121.8, 120.5, 119.6, 1189, 114.7, 113.6, 113.1, 55.3. HRMS calcd for $\mathrm{C}_{20} \mathrm{H}_{17} \mathrm{~N}_{2} \mathrm{O}:$ 301.1341. Found: 301.1353 .

\section{3-(2-Methoxyphenyl)-1-phenylimidazo[1,5-a]pyridine (3h)}

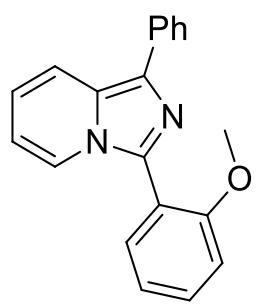

(Eluent: 4\% EtOAc/hexane); $45 \%$ yield (27.0 mg); semi-solid; ${ }^{1} \mathrm{H}$ NMR (500 MHz, $\mathrm{CDCl}_{3}$ ) $\delta 8.06(\mathrm{~d}, J=7.5 \mathrm{~Hz}, 1 \mathrm{H}), 7.85(\mathrm{~d}, J=7.5 \mathrm{~Hz}, 2 \mathrm{H}), 7.73(\mathrm{~d}, J=9.0 \mathrm{~Hz}, 1 \mathrm{H}), 7.66(\mathrm{~d}, J=8.5$ $\mathrm{Hz}, 2 \mathrm{H}), 7.38(\mathrm{t}, J=8.0 \mathrm{~Hz}, 2 \mathrm{H}), 7.21-7.16(\mathrm{~m}, 1 \mathrm{H}), 6.97(\mathrm{~d}, J=9.0 \mathrm{~Hz}, 2 \mathrm{H}), 6.67-6.64(\mathrm{~m}$, $1 \mathrm{H}), 6.45(\mathrm{t}, J=7.0 \mathrm{~Hz}, 1 \mathrm{H}), 3.78(\mathrm{~s}, 3 \mathrm{H}) .{ }^{13} \mathrm{C} \mathrm{NMR}\left(125 \mathrm{MHz}, \mathrm{CDCl}_{3}\right) \delta 159.9,138.0,135.0$, $131.5,129.7,128.6,127.2,126.7,126.3,122.5,121.6,119.3,119.0,114.4,112.9,55.3$. HRMS calcd for $\mathrm{C}_{20} \mathrm{H}_{17} \mathrm{~N}_{2} \mathrm{O}: 301.1341$. Found: 301.1353 .

3-(4-Chlorophenyl)-1-phenylimidazo[1,5-a]pyridine (3i) 


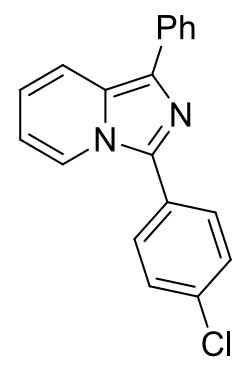

(Eluent: 4\% EtOAc/hexane); $60 \%$ yield $(36.5 \mathrm{mg})$; semi-solid; ${ }^{1} \mathrm{H}$ NMR $\left(500 \mathrm{MHz}, \mathrm{CDCl}_{3}\right)$ $\delta 7.83(\mathrm{~d}, J=8.0 \mathrm{~Hz}, 2 \mathrm{H}), 7.75(\mathrm{~d}, J=9.5 \mathrm{~Hz}, 1 \mathrm{H}), 7.70(\mathrm{~d}, J=8.0 \mathrm{~Hz}, 2 \mathrm{H}), 7.42-7.36(\mathrm{~m}$, $4 \mathrm{H}), 7.23(\mathrm{t}, J=7.5 \mathrm{~Hz}, 1 \mathrm{H}), 6.72-6.69(\mathrm{~m}, 1 \mathrm{H}), 6.51(\mathrm{t}, J=7.0 \mathrm{~Hz}, 1 \mathrm{H}) .{ }^{13} \mathrm{C} \mathrm{NMR}(125 \mathrm{MHz}$, $\left.\mathrm{CDCl}_{3}\right) \delta 136.9,134.7,134.6,132.3,129.4,129.2,128.7,128.6,127.9,126.8,126.7,121.5$, 119.8, 119.2, 113.6. HRMS calcd for $\mathrm{C}_{19} \mathrm{H}_{14} \mathrm{~N}_{2} \mathrm{Cl}$ : 305.0846. Found: 305.0857 .

\section{3-(3-Chlorophenyl)-1-phenylimidazo[1,5-a]pyridine (3j)}

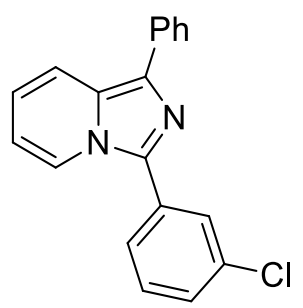

(Eluent: 4\% EtOAc/hexane); $84 \%$ yield (51.2 mg); semi-solid; ${ }^{1} \mathrm{H}$ NMR (500 MHz, $\mathrm{CDCl}_{3}$ ) $\delta 8.11(\mathrm{~d}, J=7.5 \mathrm{~Hz}, 1 \mathrm{H}), 7.82(\mathrm{~d}, J=7.5 \mathrm{~Hz}, 2 \mathrm{H}), 7.74(\mathrm{~d}, J=8.0 \mathrm{~Hz}, 2 \mathrm{H}), 7.62(\mathrm{~d}, J=7.5$ $\mathrm{Hz}, 1 \mathrm{H}), 7.38-7.29(\mathrm{~m}, 5 \mathrm{H}), 7.23(\mathrm{t}, J 7.5 \mathrm{~Hz}, 1 \mathrm{H}), 6.72(\mathrm{t}, J=7.0 \mathrm{~Hz}, 1 \mathrm{H}), 6.52(\mathrm{t}, J=6.5 \mathrm{~Hz}$ 1H). ${ }^{13} \mathrm{C} \mathrm{NMR}\left(125 \mathrm{MHz}, \mathrm{CDCl}_{3}\right) \delta 136.4,134.9,134.5,132.3,131.7,130.1,128.7,128.1$, 126.7, 1260, 121.4, 119.9, 1191, 113.6. HRMS calcd for $\mathrm{C}_{19} \mathrm{H}_{14} \mathrm{~N}_{2} \mathrm{Cl}$ : 305.0846. Found: 305.0837

3-(2-Chlorophenyl)-1-phenylimidazo[1,5-a]pyridine (3k) 


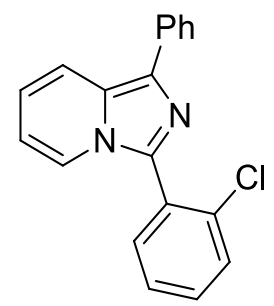

(Eluent: 4\% EtOAc/hexane); $82 \%$ yield (50.0 g); semi-solid; ${ }^{1} \mathrm{H}$ NMR (500 MHz, $\left.\mathrm{CDCl}_{3}\right) \delta$ $7.96(\mathrm{~d}, J=8.0 \mathrm{~Hz}, 2 \mathrm{H}), 7.88(\mathrm{~d}, J=9.5 \mathrm{~Hz}, 1 \mathrm{H}), 7.67(\mathrm{t}, J=5.0 \mathrm{~Hz}, 1 \mathrm{H}), 7.59(\mathrm{~d}, J=7.0 \mathrm{~Hz}$, 1H), 7,55 (d, $J=7.5 \mathrm{~Hz}, 1 \mathrm{H}), 7.47-7.42(\mathrm{~m}, 4 \mathrm{H}), 7.30-7.24(\mathrm{~m}, 1 \mathrm{H}), 6.84-6.81(\mathrm{~m}, 1 \mathrm{H}), 6.60$ $(\mathrm{t}, J=7.0 \mathrm{~Hz}, 1 \mathrm{H}) .{ }^{13} \mathrm{C} \mathrm{NMR}\left(125 \mathrm{MHz}, \mathrm{CDCl}_{3}\right) \delta 135.6,134.8,134.3,133.3,131.5,130.8$, 129.8, 129.2, 128.6, 127.2, 126.6, 126.4, 122.5, 119.8, 118.8. HRMS calcd for $\mathrm{C}_{19} \mathrm{H}_{14} \mathrm{~N}_{2} \mathrm{Cl}$ : 305.0846. Found: 305.0853.

\section{3-(4-Fluorophenyl)-1-phenylimidazo[1,5-a]pyridine (31)}

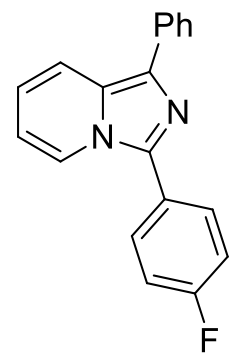

(Eluent: 4\% EtOAc/hexane); $87 \%$ yield $(50.0 \mathrm{mg})$; semi-solid; ${ }^{1} \mathrm{H}$ NMR $\left(500 \mathrm{MHz}, \mathrm{CDCl}_{3}\right)$ $\delta 8.05(\mathrm{~d}, J=7.0 \mathrm{~Hz}, 1 \mathrm{H}), 7.84(\mathrm{~d}, J=8.0 \mathrm{~Hz}, 2 \mathrm{H}), 7.74-7.69(\mathrm{~m}, 3 \mathrm{H}), 7.39(\mathrm{t}, J=7.5 \mathrm{~Hz}, 2 \mathrm{H})$, $7.22(\mathrm{t}, J=7.5 \mathrm{~Hz}, 1 \mathrm{H}), 7.16-7.11(\mathrm{~m}, 2 \mathrm{H}), 6.70(\mathrm{t}, J=6.5 \mathrm{~Hz}, 1 \mathrm{H}), 6.49(\mathrm{t}, J=6.5 \mathrm{~Hz}, 1 \mathrm{H})$. ${ }^{13} \mathrm{C}$ NMR $\left(125 \mathrm{MHz}, \mathrm{CDCl}_{3}\right) \delta 163.9(\mathrm{~d}, J=247.6 \mathrm{~Hz}), 137.1,134.8,132.0,130.3(\mathrm{~d}, J=7.8$ $\mathrm{Hz}$ ), 128.7, 127.6, 126.7, 126.6, 126.3, 121.4, 119.7, 119.1, 116.2 (d, $J=21.5 \mathrm{~Hz}), 113.4$. HRMS calcd for $\mathrm{C}_{19} \mathrm{H}_{14} \mathrm{~N}_{2} \mathrm{~F}$ : 289.1141. Found: 289.1122 .

\section{3-(3-Fluorophenyl)-1-phenylimidazo[1,5-a]pyridine (3m)}




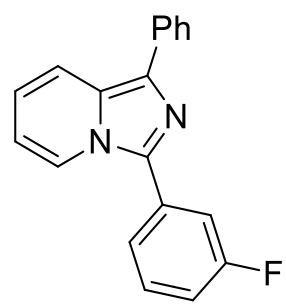

(Eluent: 4\% EtOAc/hexane); $85 \%$ yield (49.0 mg); semi-solid; ${ }^{1} \mathrm{H}$ NMR (500 MHz, $\mathrm{CDCl}_{3}$ ) $\delta 8.11-8.10(\mathrm{~m}, 1 \mathrm{H}), 7.82-7.81(\mathrm{~m}, 2 \mathrm{H}), 7.73-7.71(\mathrm{~m}, 1 \mathrm{H}), 7.52-7.50(\mathrm{~m}, 1 \mathrm{H}), 7.48-7.45(\mathrm{~m}$, 1H), 7.39-7.34 (m, 3H), 7.21-7.18 (m, 1H), 7.04-7.00 (m, 1H), 6.69-666 (m, 1H), 6.49-6.46 $(\mathrm{m}, 1 \mathrm{H}),{ }^{13} \mathrm{C} \mathrm{NMR}\left(125 \mathrm{MHz}, \mathrm{CDCl}_{3}\right) \delta 163.5(\mathrm{~d}, J=245 \mathrm{~Hz}), 136.1,134.2,131.8,131.7$, $131.6,130.1(\mathrm{~d}, J=8.2 \mathrm{~Hz}), 128.2,127.5,126.3,126.2,123.1,121.1,119.5,118.7,115.2(\mathrm{~d}$, $J=20.8 \mathrm{~Hz}), 114.8(\mathrm{~d}, J=22.6 \mathrm{~Hz}), 113.1$. HRMS calcd for $\mathrm{C}_{19} \mathrm{H}_{14} \mathrm{~N}_{2} \mathrm{~F}$ : 289.1141. Found: 289.1141

\section{3-(2-fluorophenyl)-1-phenylimidazo[1,5-a]pyridine (3n)}

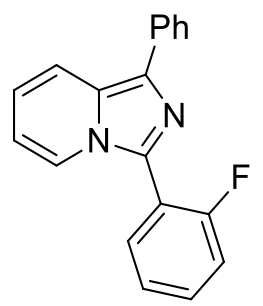

(Eluent: 4\% EtOAc/hexane); $70 \%$ yield (40.3 mg); semi-solid; ${ }^{1} \mathrm{H}$ NMR (500 MHz, $\left.\mathrm{CDCl}_{3}\right)$ $\delta 7.94(\mathrm{~d}, J=7.0 \mathrm{~Hz}, 2 \mathrm{H}), 7.85(\mathrm{~d}, J=9.0 \mathrm{~Hz}, 1 \mathrm{H}), 7.80-7.78(\mathrm{~m}, 1 \mathrm{H}), 7.75-7.73(\mathrm{~m}, 1 \mathrm{H}), 7,46$ $(\mathrm{t}, J=7.5 \mathrm{~Hz}, 3 \mathrm{H}), 7.32-727(\mathrm{~m}, 2 \mathrm{H}), 7.24-7.21(\mathrm{~m}, 1 \mathrm{H}), 6.82-6.79(\mathrm{~m}, 1 \mathrm{H}), 6,58(\mathrm{t}, J=7.5$ $\mathrm{Hz}, 1 \mathrm{H}) .{ }^{13} \mathrm{C} \mathrm{NMR}\left(125 \mathrm{MHz}, \mathrm{CDCl}_{3}\right) \delta 160.9(\mathrm{~d}, J=248 \mathrm{~Hz}), 134.8,133.3,132.5,132.2$, $131.0(\mathrm{~d}, J=7.8 \mathrm{~Hz}), 128.6,127.8,126.7,1265,124.8,122.6(\mathrm{~d}, J=5.6 \mathrm{~Hz}), 119.5,118.7$, $118.0(\mathrm{~d}, J=14.3 \mathrm{~Hz}), 116.1,115.9,112.9$. HRMS calcd for $\mathrm{C}_{19} \mathrm{H}_{14} \mathrm{~N}_{2} \mathrm{~F}$ : 289.1141. Found: 289.1158 .

\section{3-(2,6-Difluorophenyl)-1-phenylimidazo[1,5-a]pyridine (3o)}


<smiles>Fc1cccc(F)c1-c1nc(-c2ccccc2)c2ccccn12</smiles>

(Eluent: 4\% EtOAc/hexane); 90\% yield (55.0 mg); semi-solid; 1H NMR (500 MHz, CDCl3) $\delta 7.93(\mathrm{~d}, J=7.0 \mathrm{~Hz}, 2 \mathrm{H}), 7.87(\mathrm{~d}, J=8.0 \mathrm{~Hz}, 1 \mathrm{H}), 7.77(\mathrm{t}, J=6.5 \mathrm{~Hz}, 1 \mathrm{H}), 7.55-7.51(\mathrm{~m}$, 1H), $7.47(\mathrm{t}, J=8.0 \mathrm{~Hz}, 2 \mathrm{H}), 7.31(\mathrm{t}, J=7.5 \mathrm{~Hz}, 1 \mathrm{H}), 7.22-7.12(\mathrm{~m}, 1 \mathrm{H}), 6.87-6.84(\mathrm{~m}, 1 \mathrm{H})$, $6.64(\mathrm{t}, J=7.0 \mathrm{~Hz}, 1 \mathrm{H}) .13 \mathrm{C} \mathrm{NMR}(125 \mathrm{MHz}, \mathrm{CDCl} 3) \delta 159.8(\mathrm{~d}, \mathrm{~J}=117.6 \mathrm{~Hz}), 156.8(\mathrm{~d}, \mathrm{~J}=$ $243.5 \mathrm{~Hz}), 134.5,132.6,132.3,128.7,128.1,126.7,122.6$ (d, J = 6.8 Hz), 120.1, 119.4- 119.2 (m), 118.8, 118.5, 117.5-117.2 (m), 113.3. HRMS calcd for $\mathrm{C}_{19} \mathrm{H}_{13} \mathrm{~N}_{2} \mathrm{~F}_{2}$ : 307.1047 Found: 307.1056.

\section{1-phenyl-3-(4-(trifluoromethyl)phenyl)imidazo[1,5-a]pyridine (3p)}<smiles>FC(F)(F)c1ccc(-c2nc(-c3ccccc3)c3ccccn23)cc1</smiles>

(Eluent: 4\% EtOAc/hexane); $67 \%$ yield $(45.3 \mathrm{mg})$; semi-solid; ${ }^{1} \mathrm{H}$ NMR (500 MHz, $\mathrm{CDCl}_{3}$ ) $\delta 8.16(\mathrm{~d}, J=7.0 \mathrm{~Hz}, 1 \mathrm{H}), 7.89(\mathrm{~d}, J=8.5 \mathrm{~Hz}, 2 \mathrm{H}), 7.84(\mathrm{~d}, J=7.5 \mathrm{~Hz}, 2 \mathrm{H}), 7.78(\mathrm{~d}, J=9.0$ $\mathrm{Hz}, 1 \mathrm{H}), 7.69$ (d, $J=8.0 \mathrm{~Hz}, 2 \mathrm{H}), 7.40(\mathrm{t}, J=7.0 \mathrm{~Hz}, 2 \mathrm{H}), 7.24(\mathrm{t}, J=7.5 \mathrm{~Hz}, 1 \mathrm{H}), 6.75-6.72$ $(\mathrm{m}, 1 \mathrm{H}), 6.55(\mathrm{t}, J=6.5 \mathrm{~Hz}, 1 \mathrm{H}) .{ }^{13} \mathrm{C} \mathrm{NMR}\left(125 \mathrm{MHz}, \mathrm{CDCl}_{3}\right) \delta 136.4,134.6,133.6,132.8$, 130.4, 130.2, 128.8, 128.3, 128.2, 126.8, 126.0, 125.1, 121.4, 120.2, 119.3, 113.9. HRMS calcd for $\mathrm{C}_{20} \mathrm{H}_{14} \mathrm{~N}_{2} \mathrm{~F}_{3}$ : 339.1109. Found: 339.1121 . 


\section{3-(Furan-2-yl)-1-phenylimidazo[1,5-a]pyridine (3q)}

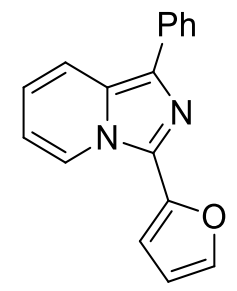

(Eluent: 4\% EtOAc/hexane); $72 \%$ yield (37.4 g); semi-solid; ${ }^{1} \mathrm{H}$ NMR $\left(500 \mathrm{MHz}, \mathrm{CDCl}_{3}\right) \delta$ $8.58(\mathrm{~d}, J=7.0 \mathrm{~Hz}, 1 \mathrm{H}), 7.84(\mathrm{~d}, J=7.0 \mathrm{~Hz}, 2 \mathrm{H}), 7.75(\mathrm{~d}, J=8.5 \mathrm{~Hz}, 1 \mathrm{H}), 7.51(\mathrm{~d}, J=2.0 \mathrm{~Hz}$ 1H), $7.40(\mathrm{t}, J=7.5 \mathrm{~Hz}, 2 \mathrm{H}), 7.24-7.18(\mathrm{~m}, 1 \mathrm{H}), 7.01(\mathrm{~d}, J=3.0 \mathrm{~Hz}, 1 \mathrm{H}), 6.75-6.72(\mathrm{~m}, 1 \mathrm{H})$, $6.61(\mathrm{t}, J=7.5 \mathrm{~Hz}, 1 \mathrm{H}), 6.53-6.52(\mathrm{~m}, 1 \mathrm{H}) .{ }^{13} \mathrm{C} \mathrm{NMR}\left(125 \mathrm{MHz}, \mathrm{CDCl}_{3}\right) \delta 146.1,142.1,134.5$, 132.3, 130.1, 128.6, 127.3, 126.9, 126.7, 123.2, 119.9, 118.9, 113.8, 111.7, 108.7. HRMS calcd for $\mathrm{C}_{16} \mathrm{H}_{13} \mathrm{~N}_{2} \mathrm{O}$ : 261.1028. Found: 261.1046.

\section{3-Cyclopropyl-1-phenylimidazo[1,5-a]pyridine (3s)}

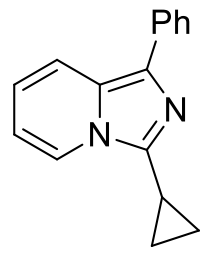

(Eluent: 4\% EtOAc/hexane); $51 \%$ yield $(24.0 \mathrm{~g})$; semi-solid; ${ }^{1} \mathrm{H}$ NMR $\left(500 \mathrm{MHz}, \mathrm{CDCl}_{3}\right) \delta$ $7.99(\mathrm{~d}, J=7.0 \mathrm{~Hz}, 1 \mathrm{H}), 7.85(\mathrm{~d}, J=7.0 \mathrm{~Hz}, 2 \mathrm{H}), 7.75(\mathrm{~d}, J=8.0 \mathrm{~Hz}, 1 \mathrm{H}), 7.43(\mathrm{t}, J=7.5 \mathrm{~Hz}$ 2H), $7.25(\mathrm{t}, J=8.0 \mathrm{~Hz}, 1 \mathrm{H}), 6.74-6.71(\mathrm{~m}, 1 \mathrm{H}), 6.59(\mathrm{t}, J=7.0 \mathrm{~Hz}, 1 \mathrm{H}), 2.07-2.01(\mathrm{~m}, 1 \mathrm{H})$, 1.14-1.09 (m, 4H). ${ }^{13} \mathrm{C}$ NMR (125 MHz, $\left.\mathrm{CDCl}_{3}\right) \delta 139.4,135.0,130.9,129.7,129.4,129.0$, 128.6, 126.8, 126.6, 126.4, 126.1, 121.1, 119.0, 118.9, 112.3. 6.6, 6.0. HRMS calcd for $\mathrm{C}_{16} \mathrm{H}_{15} \mathrm{~N}_{2}: 235.1235$. Found: 235.1238 .

Ethyl 3-phenylimidazo[1,5-a]pyridine-1-carboxylate (3u) 


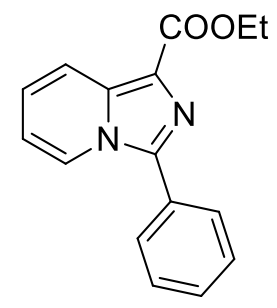

(Eluent: Dichloromethane); $51 \%$ yield $(27 \mathrm{mg})$; solid; ${ }^{1} \mathrm{H}$ NMR $\left(500 \mathrm{MHz}, \mathrm{CDCl}_{3}\right) \delta 8.30$ $8.24(\mathrm{~m}, 2 \mathrm{H}), 7,80(\mathrm{~d}, J=7.0 \mathrm{~Hz}, 2 \mathrm{H}), 7.54-7,48(\mathrm{~m}, 3 \mathrm{H}), 7.14-7.11(\mathrm{~m}, 1 \mathrm{H}), 6.77(\mathrm{t}, J=6.5$ $\mathrm{Hz}, 1 \mathrm{H}), 4.50(\mathrm{q}, J=7.0 \mathrm{~Hz}, 2 \mathrm{H}), 1.46(\mathrm{t}, J=7.0 \mathrm{~Hz}, 3 \mathrm{H}) .{ }^{13} \mathrm{C} \mathrm{NMR}\left(125 \mathrm{MHz}, \mathrm{CDCl}_{3}\right) \delta$ $163.5,139.1,135.3,129.4,128.9,128.7,124.1,122.4,120.0,114.3,60.3$. HRMS calcd for $\mathrm{C}_{16} \mathrm{H}_{15} \mathrm{~N}_{2} \mathrm{O}_{2}: 267.1134$. Found: 267.1130.

Ethyl 3-(4-methoxyphenyl)imidazo[1,5-a]pyridine-1-carboxylate(3v)

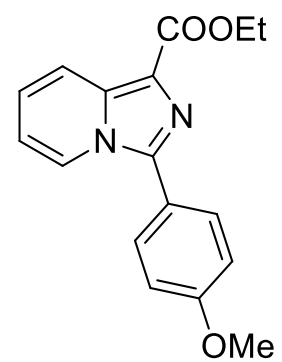

(Eluent: 20\% EtOAc/hexane); 95\% yield (35.2 mg); solid; ${ }^{1} \mathrm{H} \mathrm{NMR}\left(500 \mathrm{MHz}, \mathrm{CDCl}_{3}\right) \delta 8.23$ $(\mathrm{t}, J=8.0 \mathrm{~Hz}, 2 \mathrm{H}), 7.71(\mathrm{~d}, J=8.5 \mathrm{~Hz}, 2 \mathrm{H}), 7.10(\mathrm{t}, J=8.0 \mathrm{~Hz}, 1 \mathrm{H}), 7.05(\mathrm{~d}, J=7.5 \mathrm{~Hz}, 2 \mathrm{H})$, $6.75(\mathrm{t}, J=7.0 \mathrm{~Hz}, 1 \mathrm{H}), 4,49(\mathrm{q}, J=7.0 \mathrm{~Hz}, 2 \mathrm{H}), 3.87(\mathrm{~s}, 3 \mathrm{H}), 1.45(\mathrm{t}, J=7.0 \mathrm{~Hz}, 3 \mathrm{H}),{ }^{13} \mathrm{C} \mathrm{NMR}$ $\left(125 \mathrm{MHz}, \mathrm{CDCl}_{3}\right) \delta 163.6,160.4,139.1,135.2,130.2,126.3,123.9,122.4,121.4,119.9$, 114.3, 114.1, 60.2, 55.3, 14.6. HRMS calcd for $\mathrm{C}_{17} \mathrm{H}_{17} \mathrm{~N}_{2} \mathrm{O}_{3}: 297.1239$. Found: 297.1249.

\section{Ethyl 3-(4-fluorophenyl)imidazo[1,5-a]pyridine-1-carboxylate(3w)}




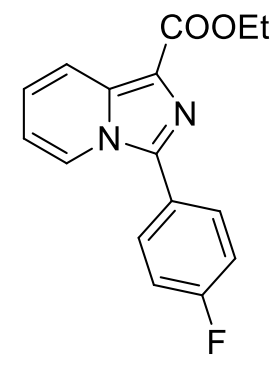

(Eluent: 20\% EtOAc/hexane); $47 \%$ yield $(26.5 \mathrm{mg})$; solid; ${ }^{1} \mathrm{H} \mathrm{NMR}\left(500 \mathrm{MHz}, \mathrm{CDCl}_{3}\right) \delta 8.23$ $(\mathrm{t}, J=7.5 \mathrm{~Hz}, 2 \mathrm{H}), 7.78-7.75(\mathrm{~m}, 2 \mathrm{H}), 7.22(\mathrm{t}, J=7.5 \mathrm{~Hz}, 2 \mathrm{H}), 7.13(\mathrm{t}, J=7.0 \mathrm{~Hz}, 1 \mathrm{H}), 6,79(\mathrm{t}$, $J=6.5 \mathrm{~Hz}, 1 \mathrm{H}), 4.50(\mathrm{q}, J=7.0 \mathrm{~Hz}, 2 \mathrm{H}), 1.46(\mathrm{t}, J=7.0 \mathrm{~Hz}, 3 \mathrm{H}) .{ }^{13} \mathrm{C} \mathrm{NMR}\left(125 \mathrm{MHz}, \mathrm{CDCl}_{3}\right)$ $\delta$ 1642. $(\mathrm{d}, J=248.8 \mathrm{~Hz}), 163.4,138.0,135.5,130.7(\mathrm{~d}, J=8.1 \mathrm{~Hz}), 125.3,124.1,122.1,121.7$, 120.0, $116.1(\mathrm{~d}, J=21.7 \mathrm{~Hz}), 114.4,60.3,14.5$. HRMS calcd for $\mathrm{C}_{16} \mathrm{H}_{14} \mathrm{~N}_{2} \mathrm{O} 2 \mathrm{~F}: 285.1039$. Found: 285.1032.

\section{General procedure for the synthesis of imidazo[1,5-a]pyridine (5a) from amino acids}

General procedure for the synthesis of imidazo[1,5-a]pyridine (5a): To a reaction tube equipped with a magnetic stir bar was added 3-phenyl-[1,2,3]triazolo[1,5-a]pyridine $\mathbf{1 a}$ (39.0mg, $0.2 \mathrm{mmol})$, D-Phenylglycine 4 (90.6mg, $0.6 \mathrm{mmol})$, and copper iodide (0.08 mmol, $40 \mathrm{~mol} \%$ ) and $1.0 \mathrm{~mL}$ of 1,2-dichlorobenzne. The mixture was heated in an oil bath at $150{ }^{\circ} \mathrm{C}$ in a closed tube. The reaction was monitored by TLC, after completion of the reaction, it was allowed to attain room temperature. Then the mixture was poured into $30 \mathrm{~mL}$ of sodium chloride solution. The product was extracted with EtOAc $(15 \mathrm{~mL} \mathrm{X} \mathrm{3)}$ and dried over anhydrous $\mathrm{Na}_{2} \mathrm{SO}_{4}$. Removal of the solvent under reduced pressure the left out crude residue was purified by column chromatography using silica gel (4\% EtOAc/hexane) to afford $\mathbf{5 a}$ (43.2 mg; $80 \%$ yield). 
<smiles>Oc1ccc(-c2nc(-c3ccccc3)c3ccccn23)cc1</smiles>

(Eluent: 20\% EtOAc/hexane); $26 \%$ yield (14.88 mg); semi-solid; ${ }^{1} \mathrm{H}$ NMR (500 MHz, DMSOd 6$) \delta 9.89(\mathrm{~s}, 1 \mathrm{H}), 8.35(\mathrm{~d}, J=7.0 \mathrm{~Hz}, 1 \mathrm{H}), 7.97-7.92(\mathrm{~m}, 3 \mathrm{H}), 7.68(\mathrm{~d}, J=8.5 \mathrm{~Hz}, 2 \mathrm{H}), 7.46$ $(\mathrm{t}, J=7.0 \mathrm{~Hz}, 2 \mathrm{H}), 7.27(\mathrm{t}, J=7.5 \mathrm{~Hz}, 1 \mathrm{H}), 6.98(\mathrm{~d}, J=7.0 \mathrm{~Hz}, 2 \mathrm{H}), 6.93-6.89(\mathrm{~m}, 1 \mathrm{H}), 6.74$ $(\mathrm{t}, J=7.0 \mathrm{~Hz}, 1 \mathrm{H}) .{ }^{13} \mathrm{C} \mathrm{NMR}\left(125 \mathrm{MHz}, \mathrm{CDCl}_{3}\right) \delta 157.9,137.8,134.8,130.5,129.7,129.5$, 129.3, 128.6, 126.7, 126.0, 125.8, 122.3, 120.4, 120.3, 118.4, 115.7, 114.9, 113.3. HRMS calcd for $\mathrm{C}_{19} \mathrm{H}_{15} \mathrm{~N}_{2} \mathrm{O}: 287.1184$. Found: 287.1184.

\section{3-Methyl-1-phenylimidazo[1,5-a]pyridine (5f)}

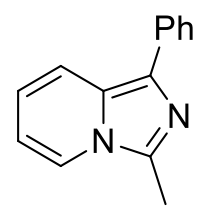

(Eluent: 20\% EtOAc/hexane); $65 \%$ yield (27.0 mg); semi-solid; ${ }^{1} \mathrm{H}$ NMR (500 MHz, $\left.\mathrm{CDCl}_{3}\right)$ $\delta 7.85(\mathrm{~d}, J=7.0 \mathrm{~Hz}, 2 \mathrm{H}), 7.76(\mathrm{~d}, J=9.0 \mathrm{~Hz}, 1 \mathrm{H}), 7.65(\mathrm{~d}, J=7.5 \mathrm{~Hz}, 1 \mathrm{H}), 7.45(\mathrm{t}, J=7.5$ $\mathrm{Hz}, 2 \mathrm{H}), 7.27(\mathrm{t}, J=6.5 \mathrm{~Hz}, 1 \mathrm{H}), 6.73-6.70(\mathrm{~m}, 1 \mathrm{H}), 6.58(\mathrm{t}, J=7.0 \mathrm{~Hz}, 1 \mathrm{H}), 2.68(\mathrm{~s}, 3 \mathrm{H}) .{ }^{13} \mathrm{C}$ $\operatorname{NMR}\left(125 \mathrm{MHz}, \mathrm{CDCl}_{3}\right) \delta 135.1,134.9,129.8,128.7,126.3,126.1,121.0,118.9,118.6,112.5$, 12.5. HRMS calcd for $\mathrm{C}_{14} \mathrm{H}_{13} \mathrm{~N}_{2} \mathrm{Cl}$ : 209.1079. Found: 209.1079.

\section{3-isobutyl-1-phenylimidazo[1,5-a]pyridine (5g)}




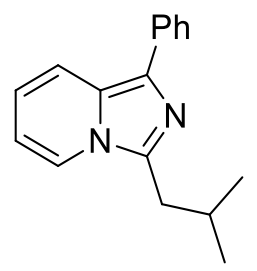

(Eluent: 15\% EtOAc/hexane); $59 \%$ yield (29.5 g); semi-solid; ${ }^{1} \mathrm{H}$ NMR $\left(500 \mathrm{MHz}, \mathrm{CDCl}_{3}\right) \delta$ $7.86(\mathrm{~d}, J=8.0 \mathrm{~Hz}, 2 \mathrm{H}), 7.75(\mathrm{t}, J=7.5 \mathrm{~Hz}, 2 \mathrm{H}), 7.44(\mathrm{t}, J=8.0 \mathrm{~Hz}, 2 \mathrm{H}), 7.26(\mathrm{t}, J=7.0 \mathrm{~Hz}$, 1H), 6.70-6.67 (m, 1H), $6.53(\mathrm{t}, J=7.0 \mathrm{~Hz}, 1 \mathrm{H}), 2.90(\mathrm{~d}, J=7.0 \mathrm{~Hz}, 2 \mathrm{H}), 2.25$ (septet, $J=7.0$ $\mathrm{Hz}, 1 \mathrm{H}), 1.02(\mathrm{~d}, J=6.5 \mathrm{~Hz}, 6 \mathrm{H}) .{ }^{13} \mathrm{C} \mathrm{NMR}\left(125 \mathrm{MHz}, \mathrm{CDCl}_{3}\right) \delta 138.4,135.2,130.1,128.6$, 126.5, 126.3, 126.0, 121.0, 118.9, 118.4, 112.3, 35.5, 27.8, 22.6. HRMS calcd for $\mathrm{C}_{17} \mathrm{H}_{19} \mathrm{~N}_{2}$ Cl Na: 251.1548. Found: 251.1555. 


\section{Copies of ${ }^{1} \mathrm{H} \&{ }^{13} \mathrm{C}$ NMR spectra}




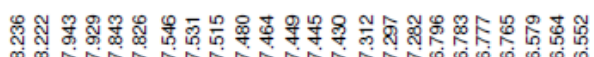

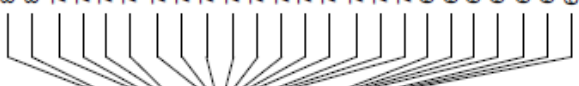

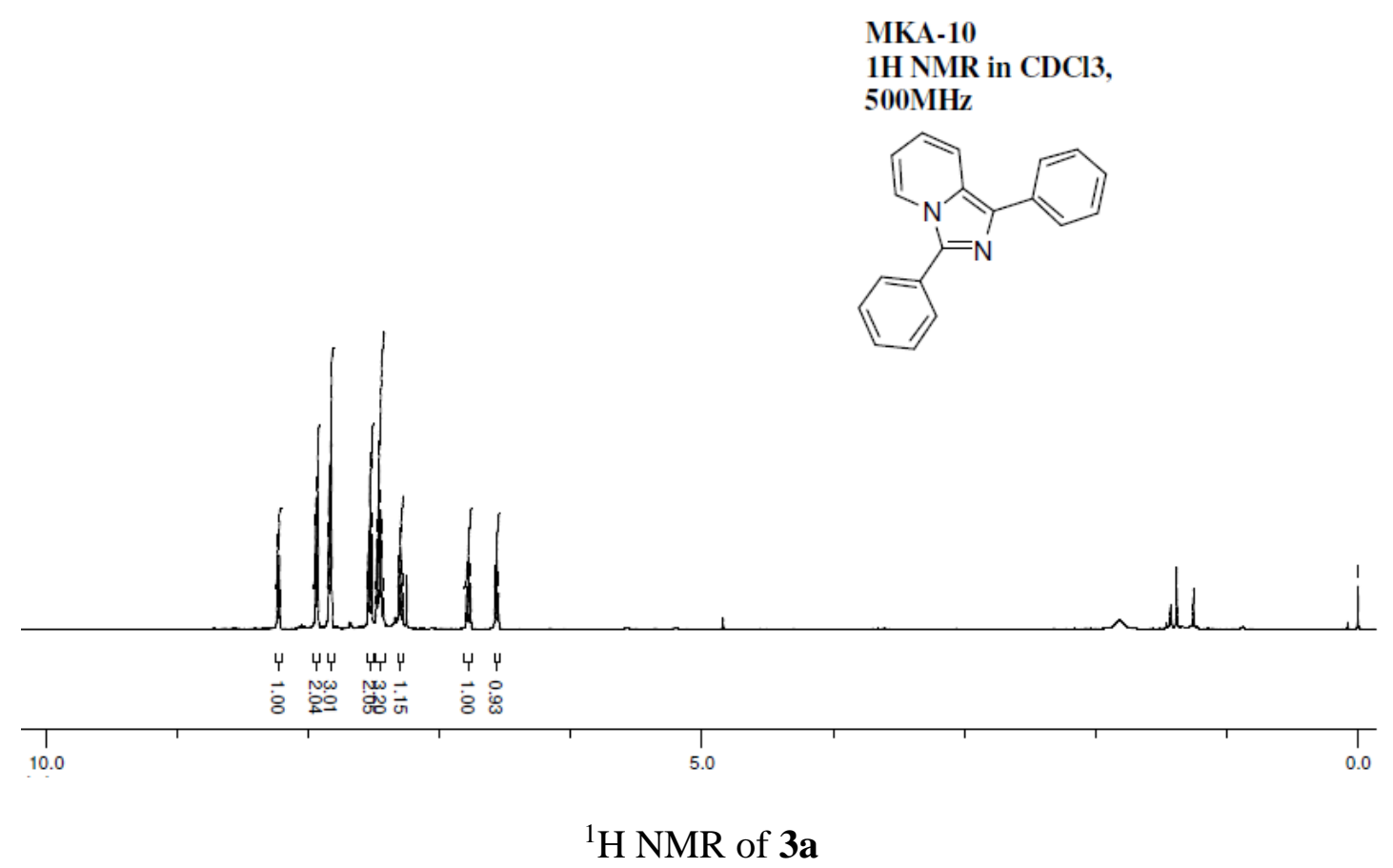

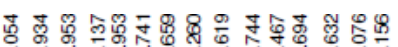

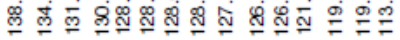

$\lfloor\lfloor u)\rfloor J\rfloor j$

蒙字是

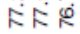

l)

MKA-10

13C NMR in CDCl3,

$500 \mathrm{MHz}$
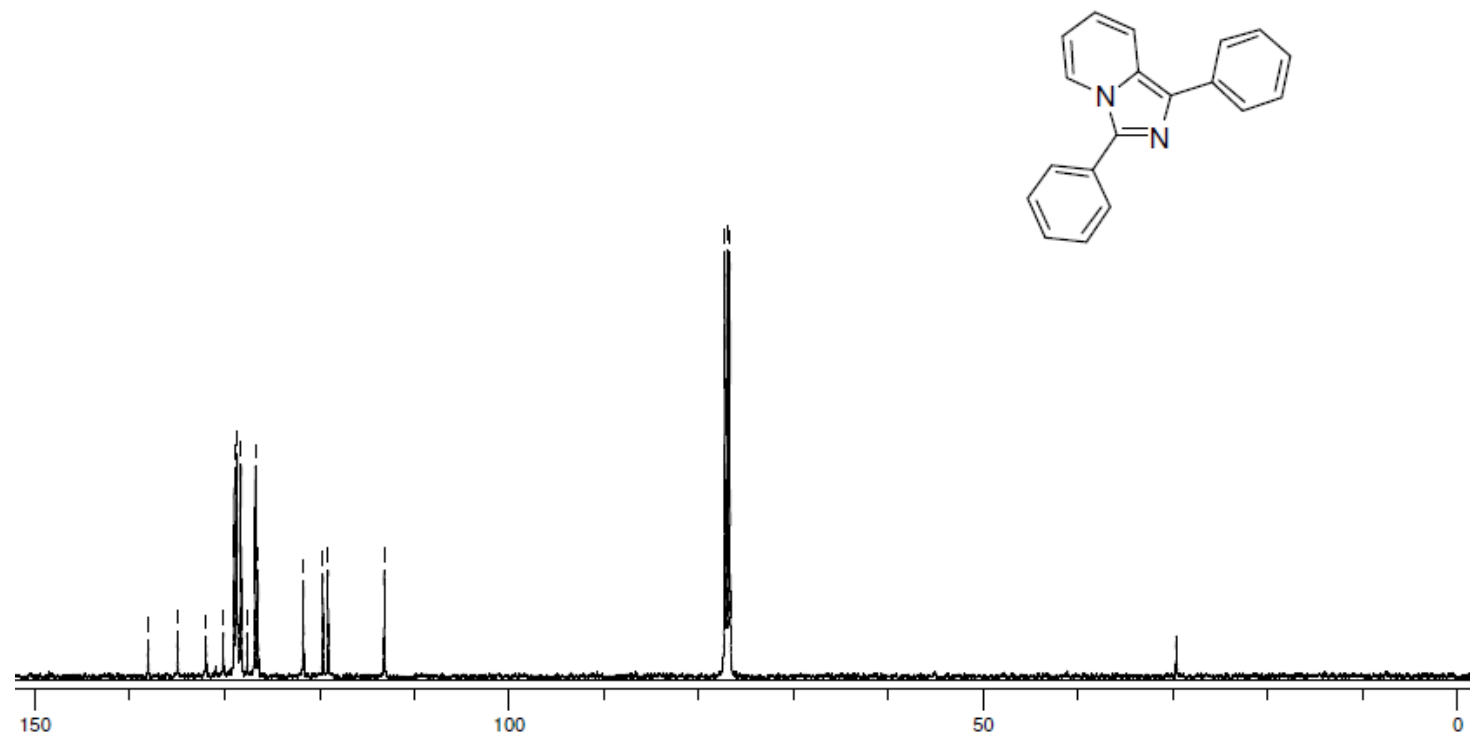

${ }^{13} \mathrm{C}$ NMR of 3a 

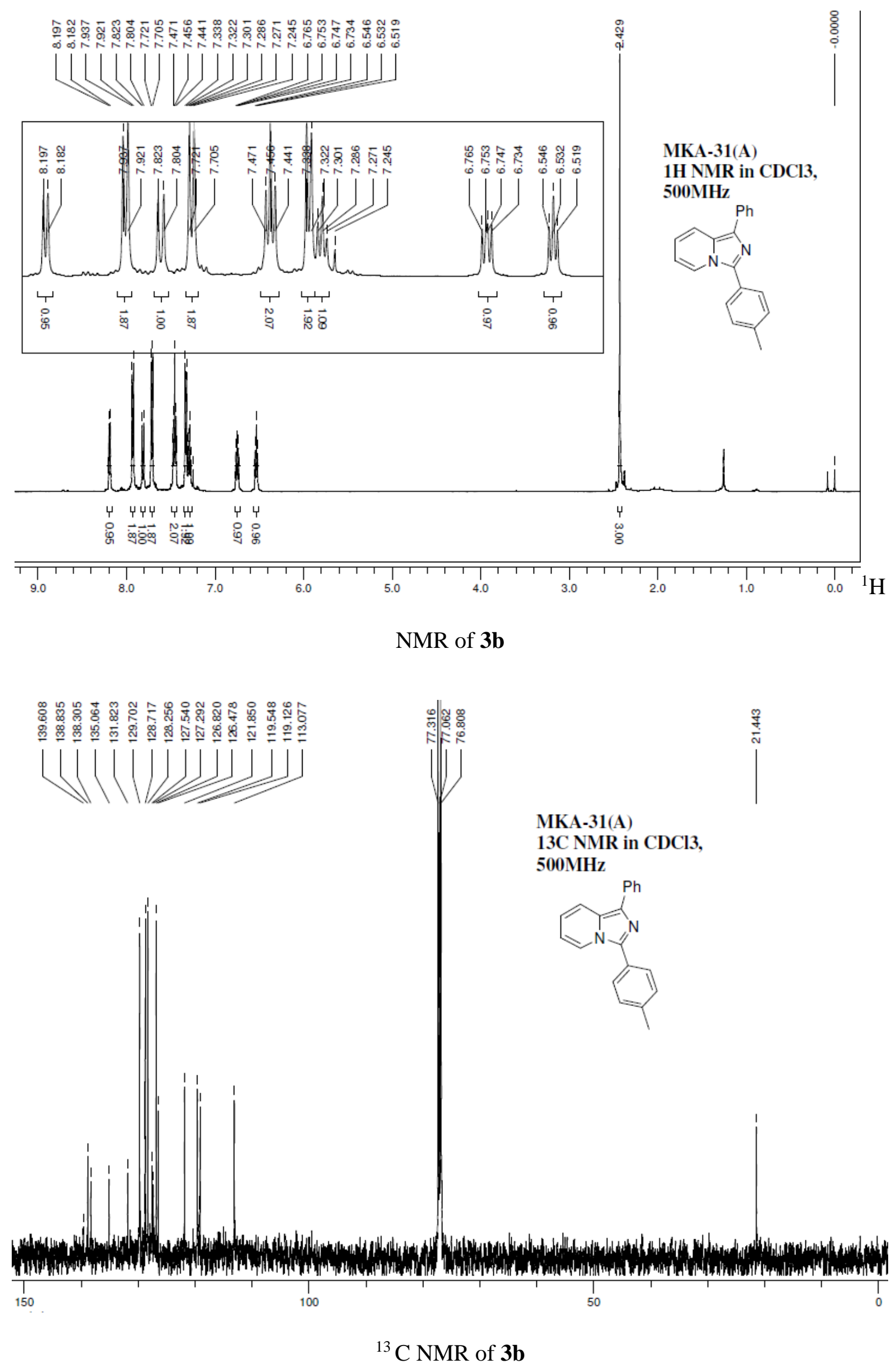


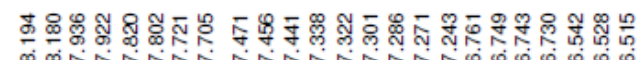

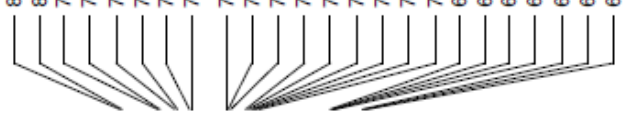

MKA-31

1H NMR in CDCl3,

$500 \mathrm{MHz}$
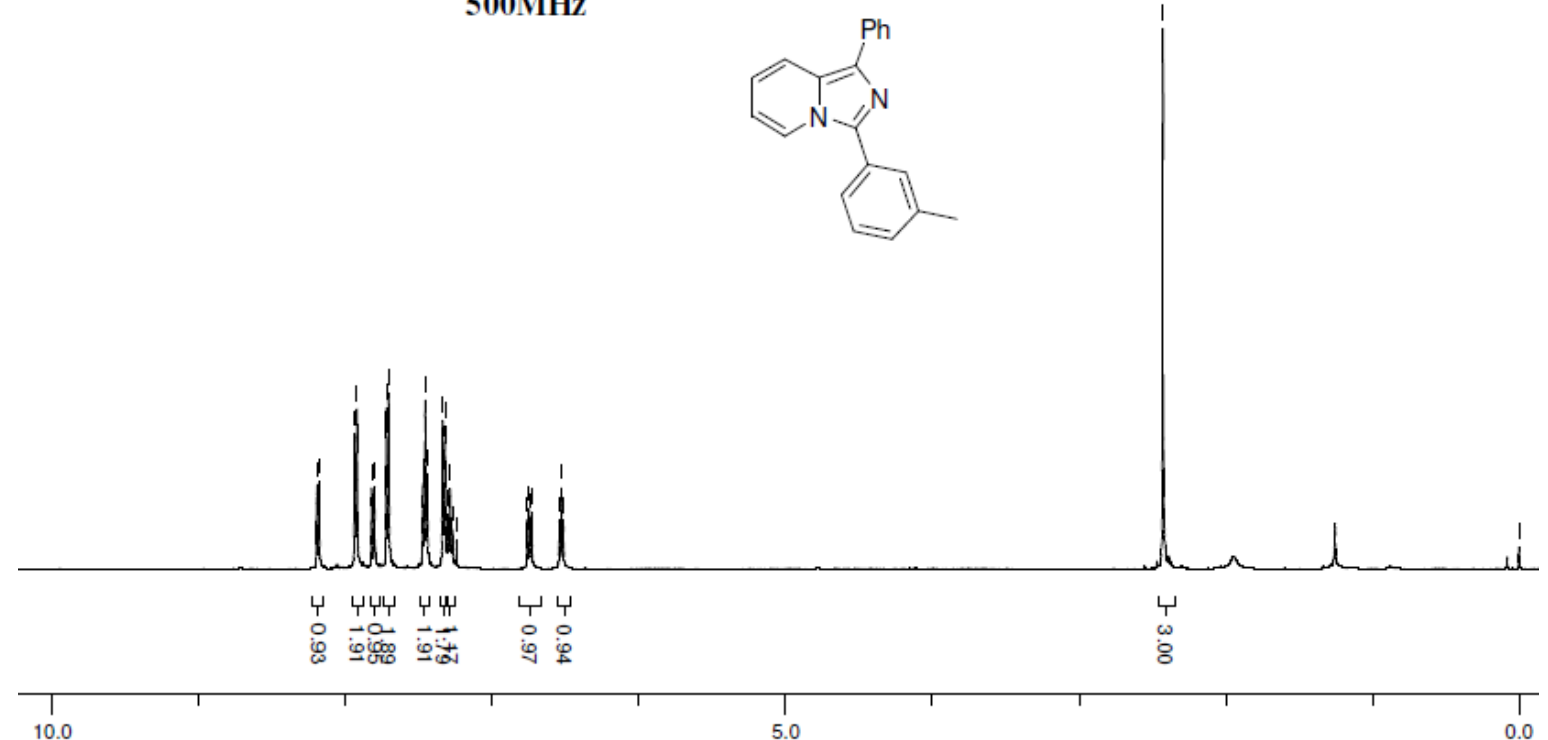

${ }^{1} \mathrm{H}$ NMR of $\mathbf{3 c}$

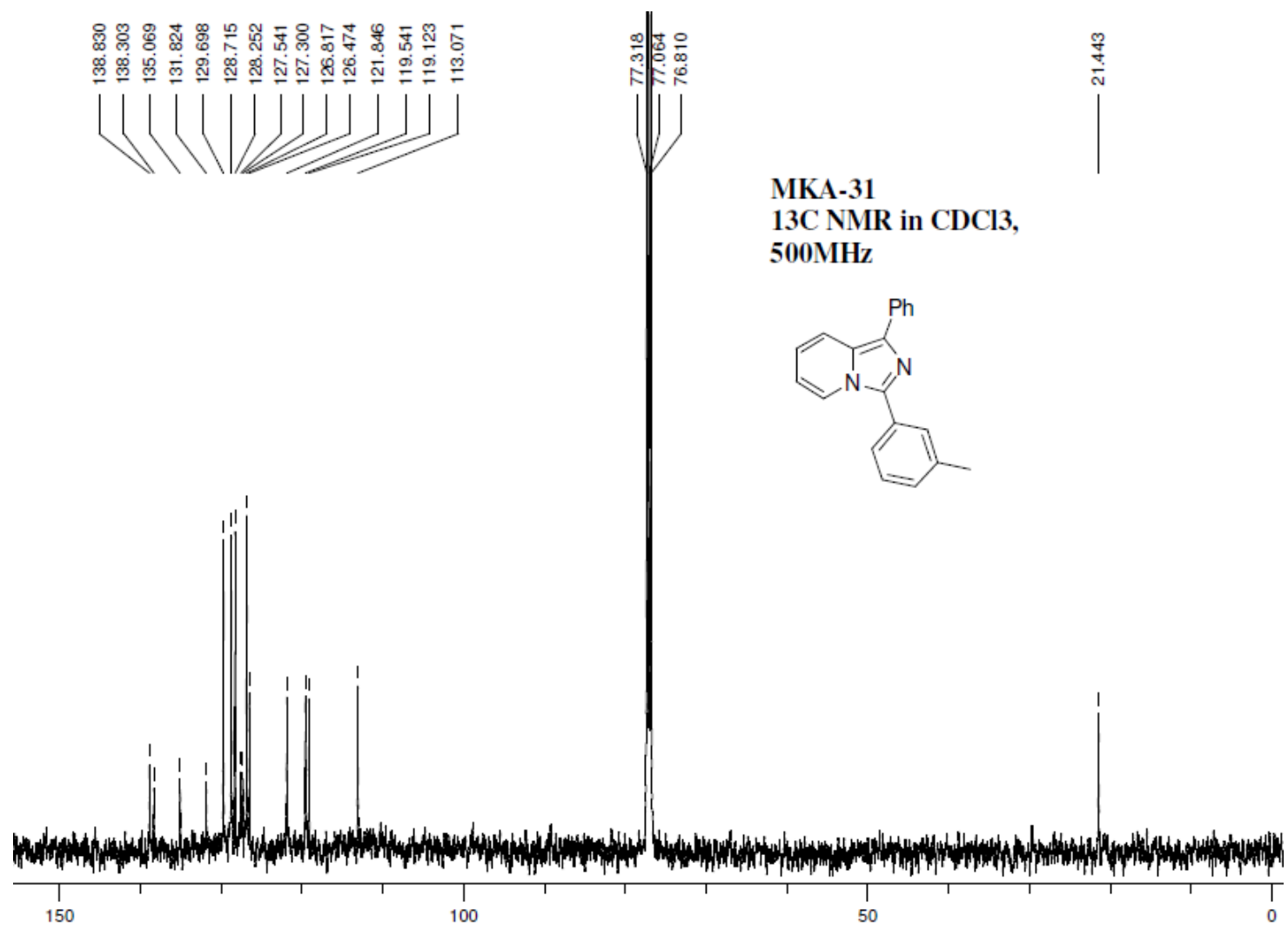

${ }^{13} \mathrm{C}$ NMR of $\mathbf{3 c}$ 


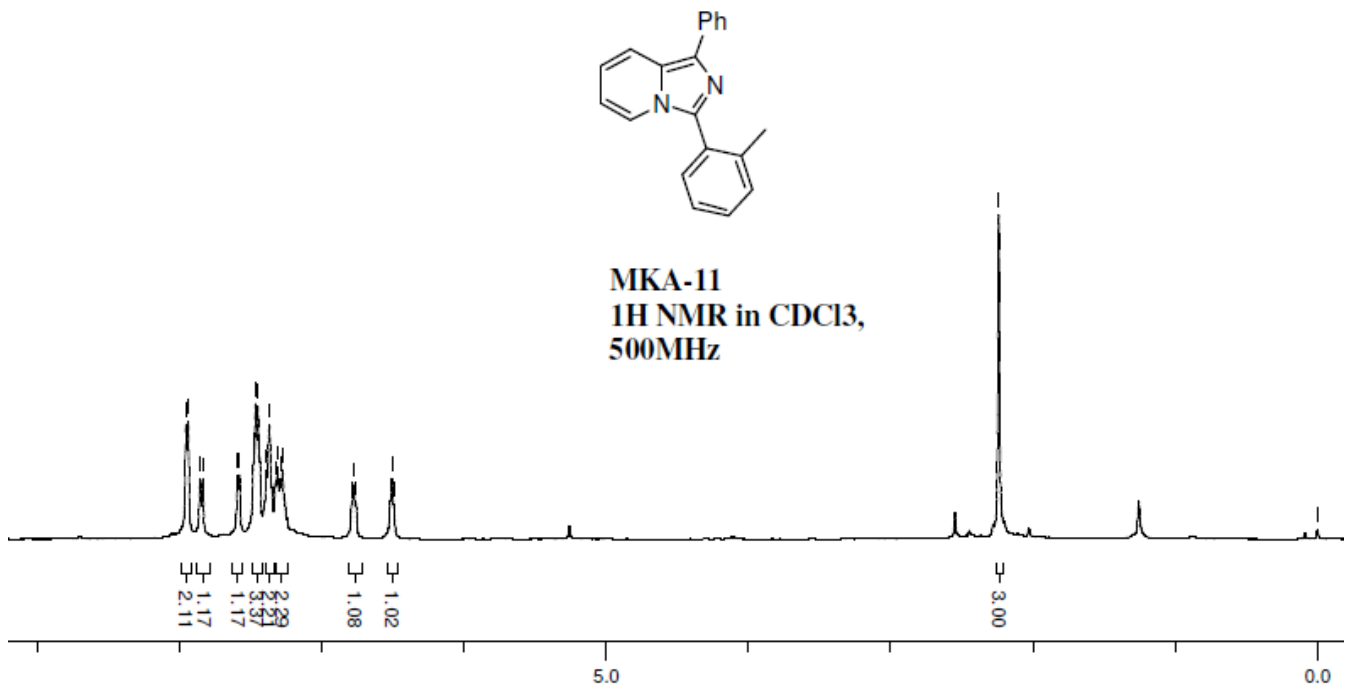

${ }^{1}$ H NMR of $\mathbf{3 d}$

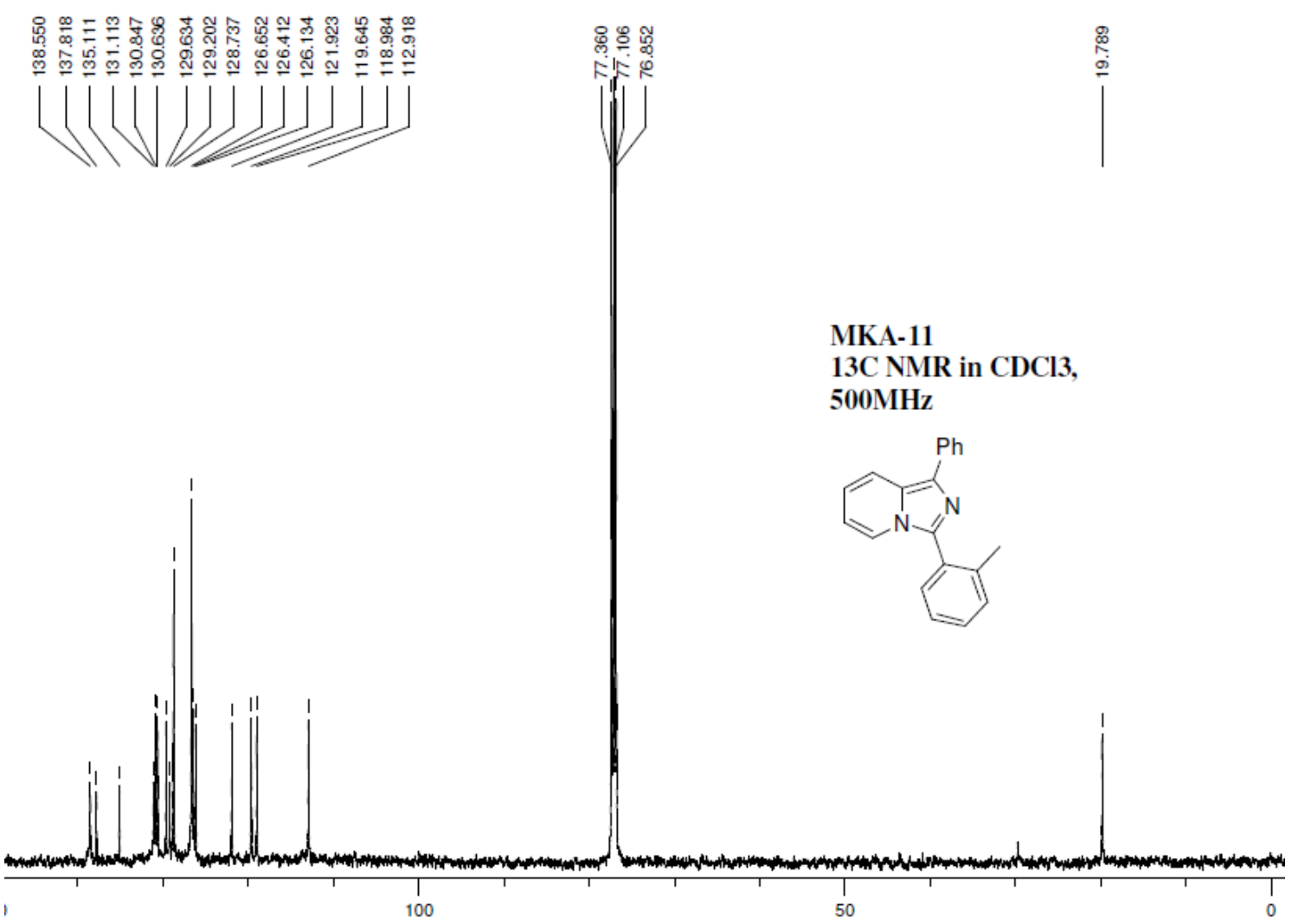

${ }^{13} \mathrm{C}$ NMR of 3d 


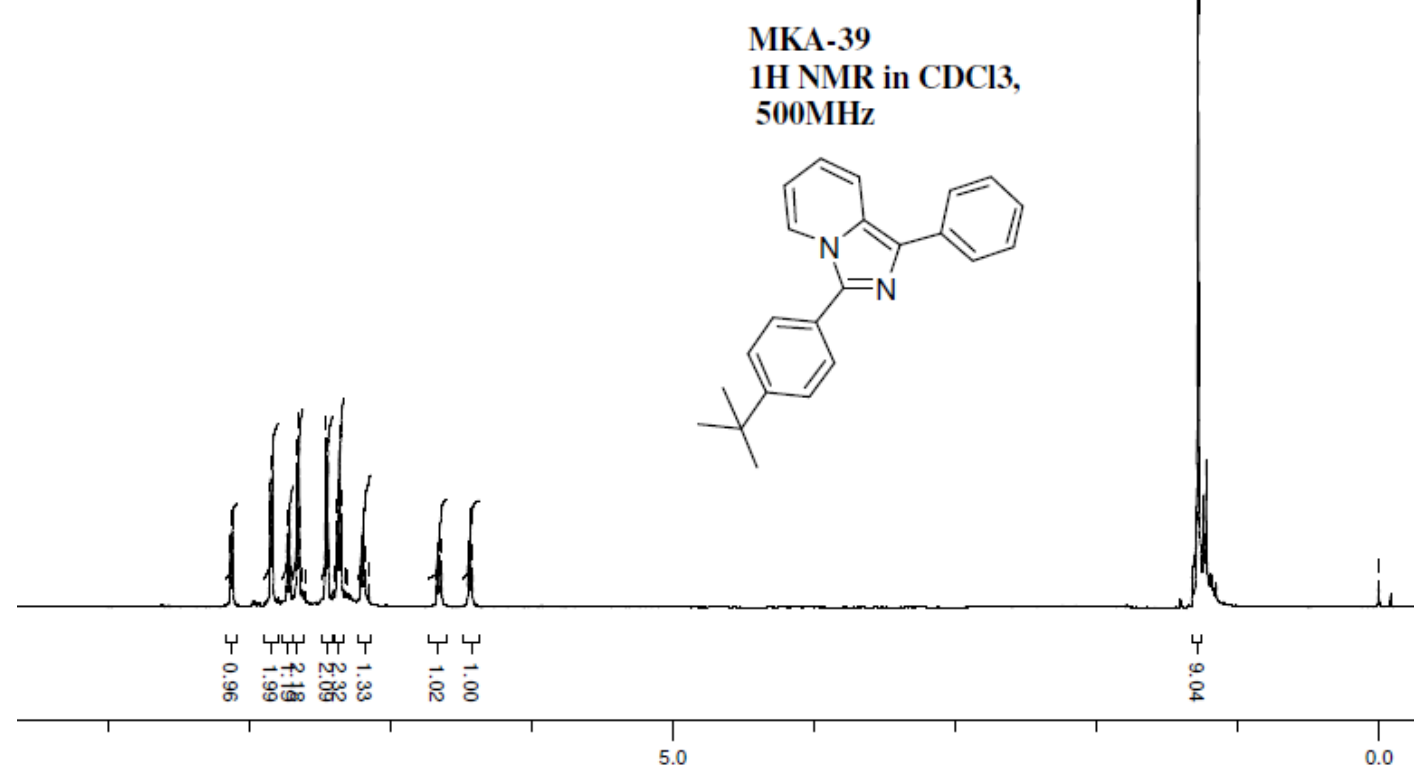

${ }^{1} \mathrm{H}$ NMR of $\mathbf{3 e}$

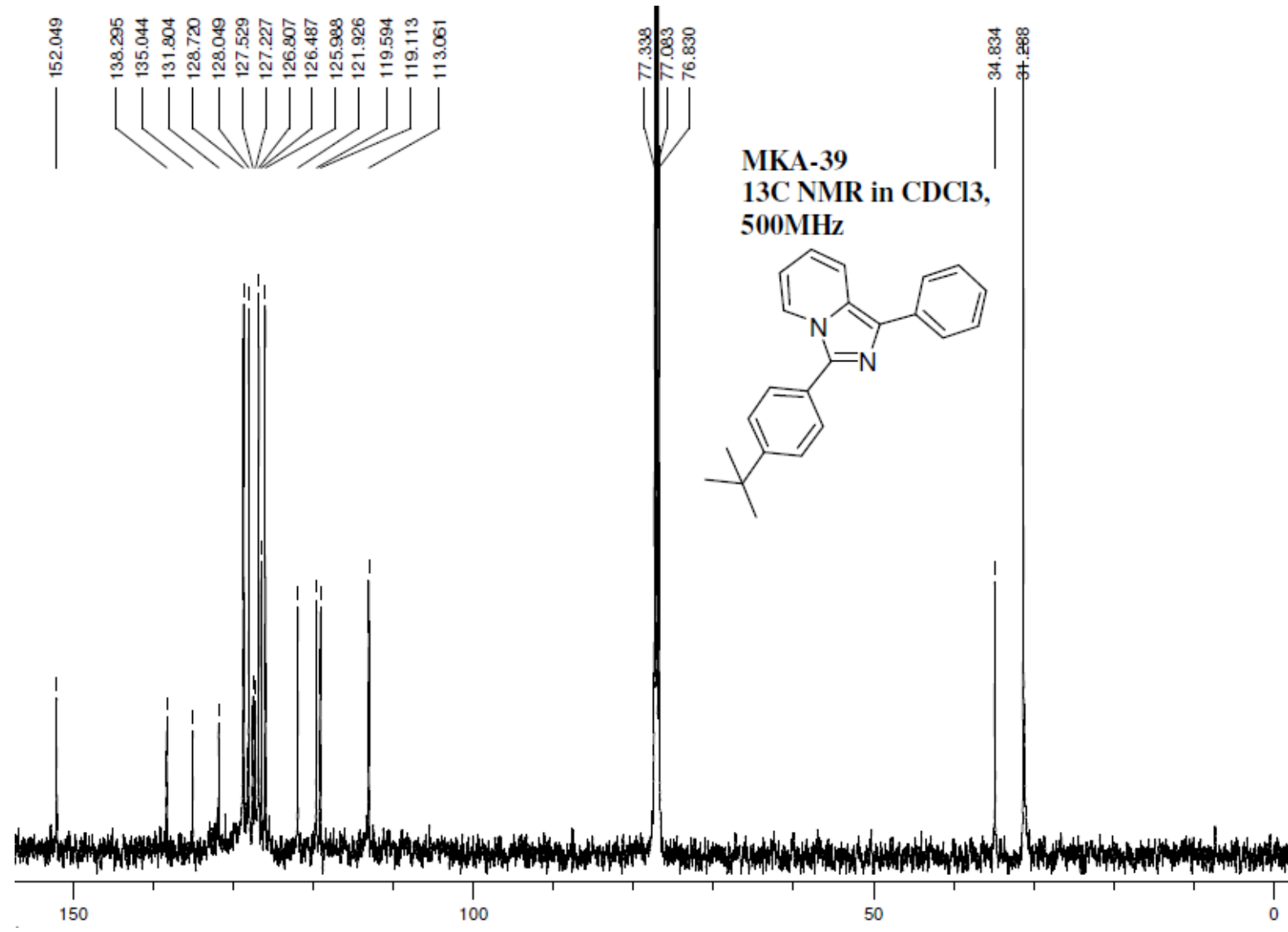

${ }^{13} \mathrm{C}$ NMR of $\mathbf{3 e}$ 


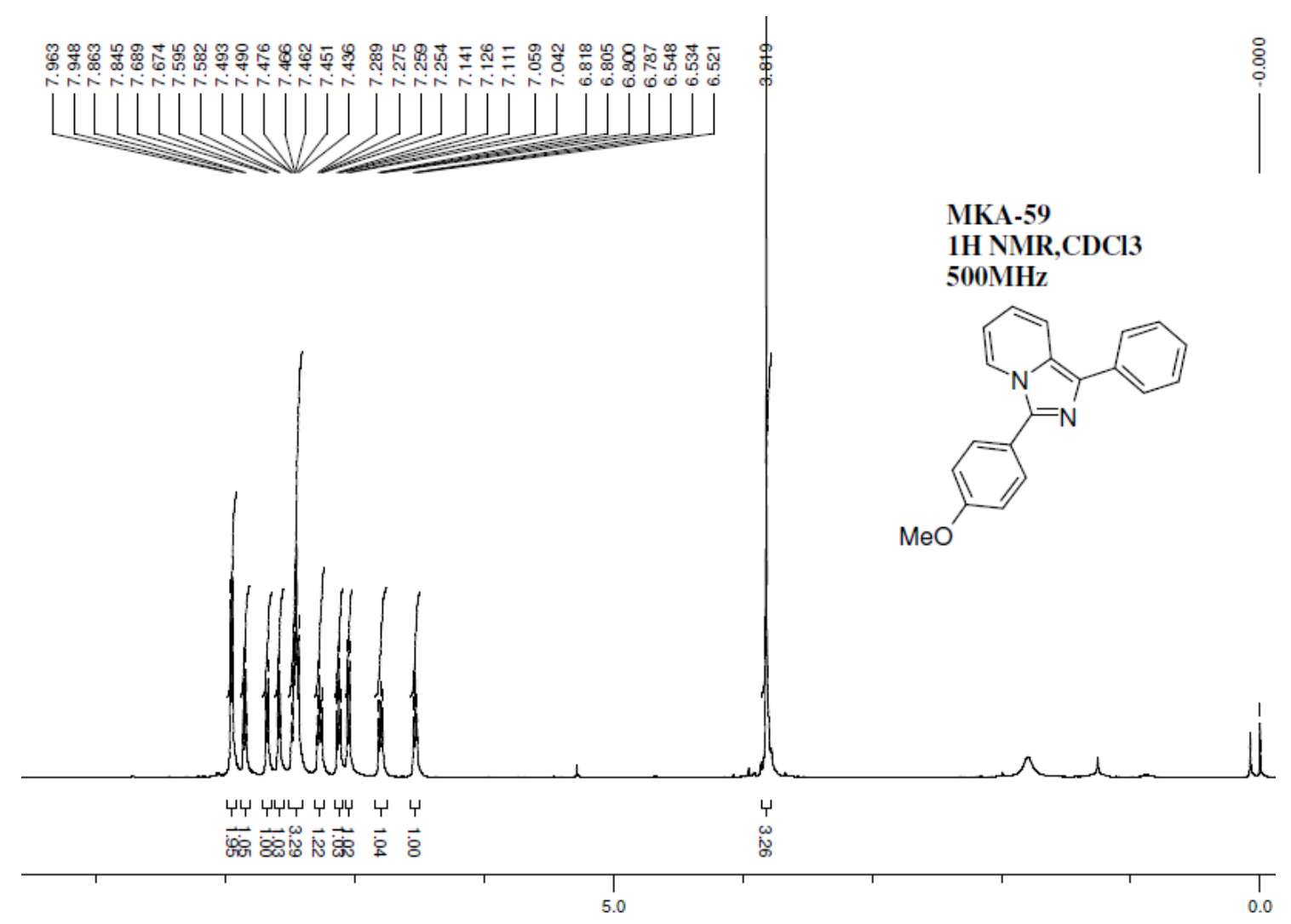

${ }^{1} \mathrm{H}$ NMR of $\mathbf{3 f}$

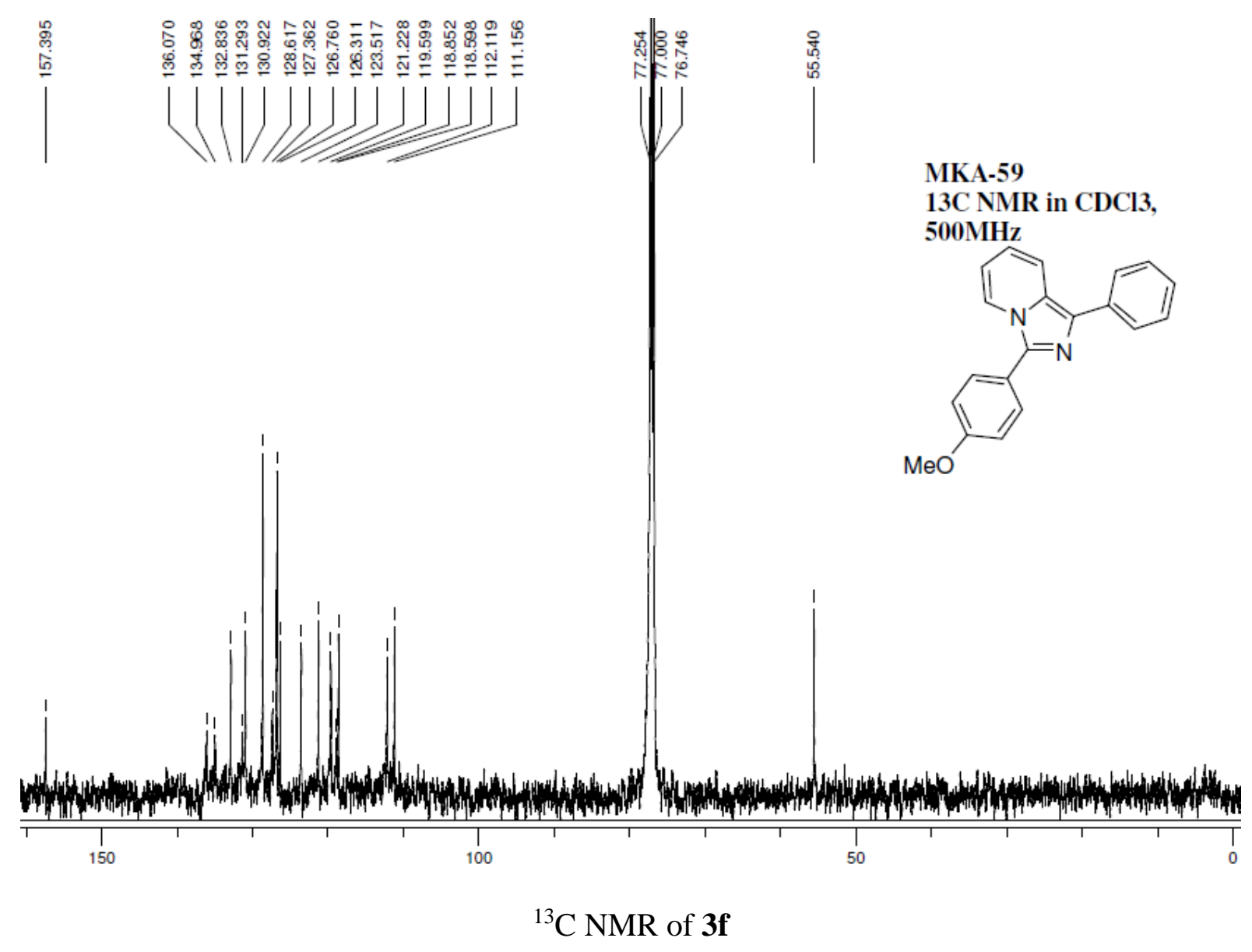



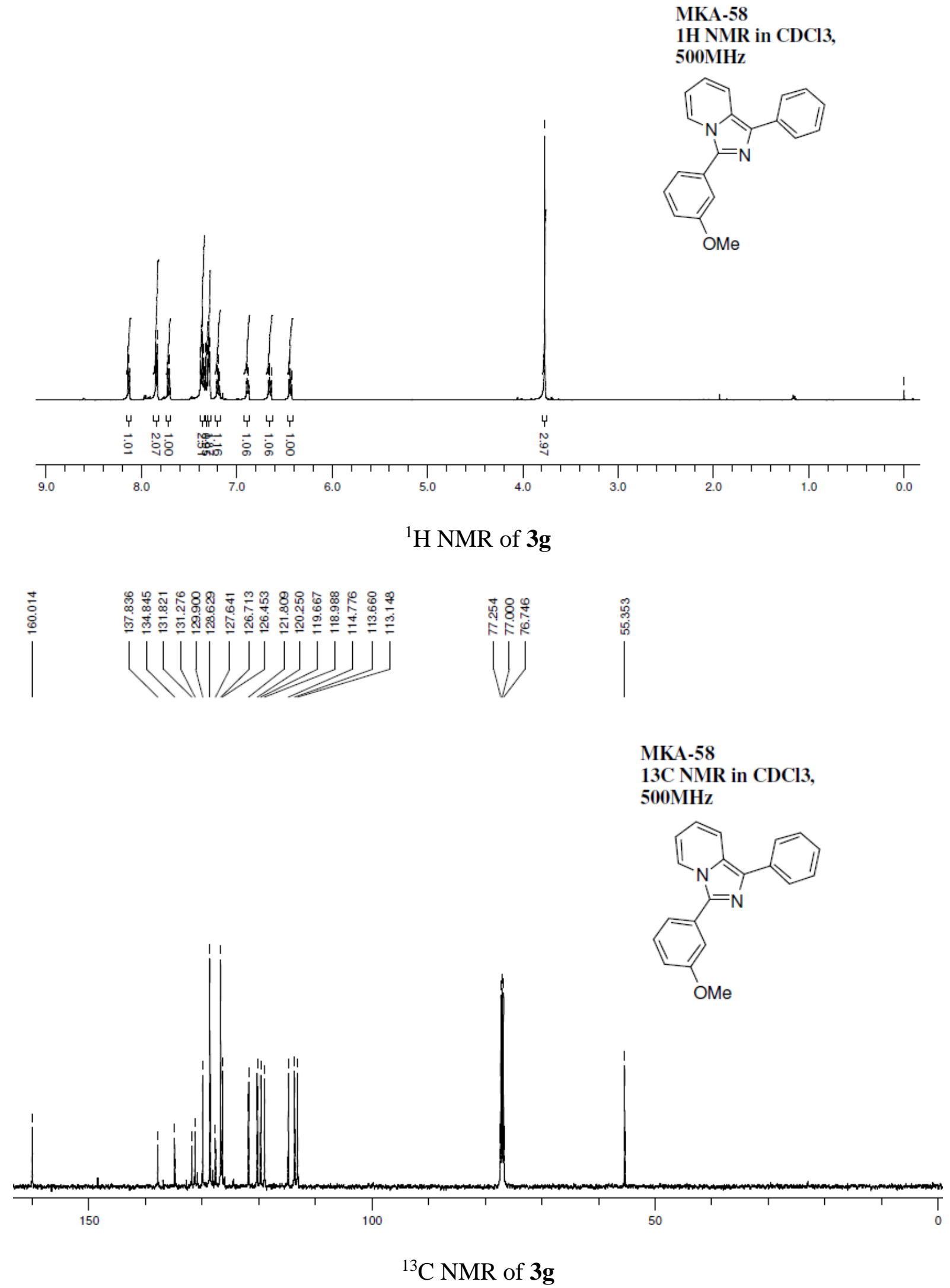
MKA-57

$1 \mathrm{H}$ NMR,CDCl3

$500 \mathrm{MHz}$
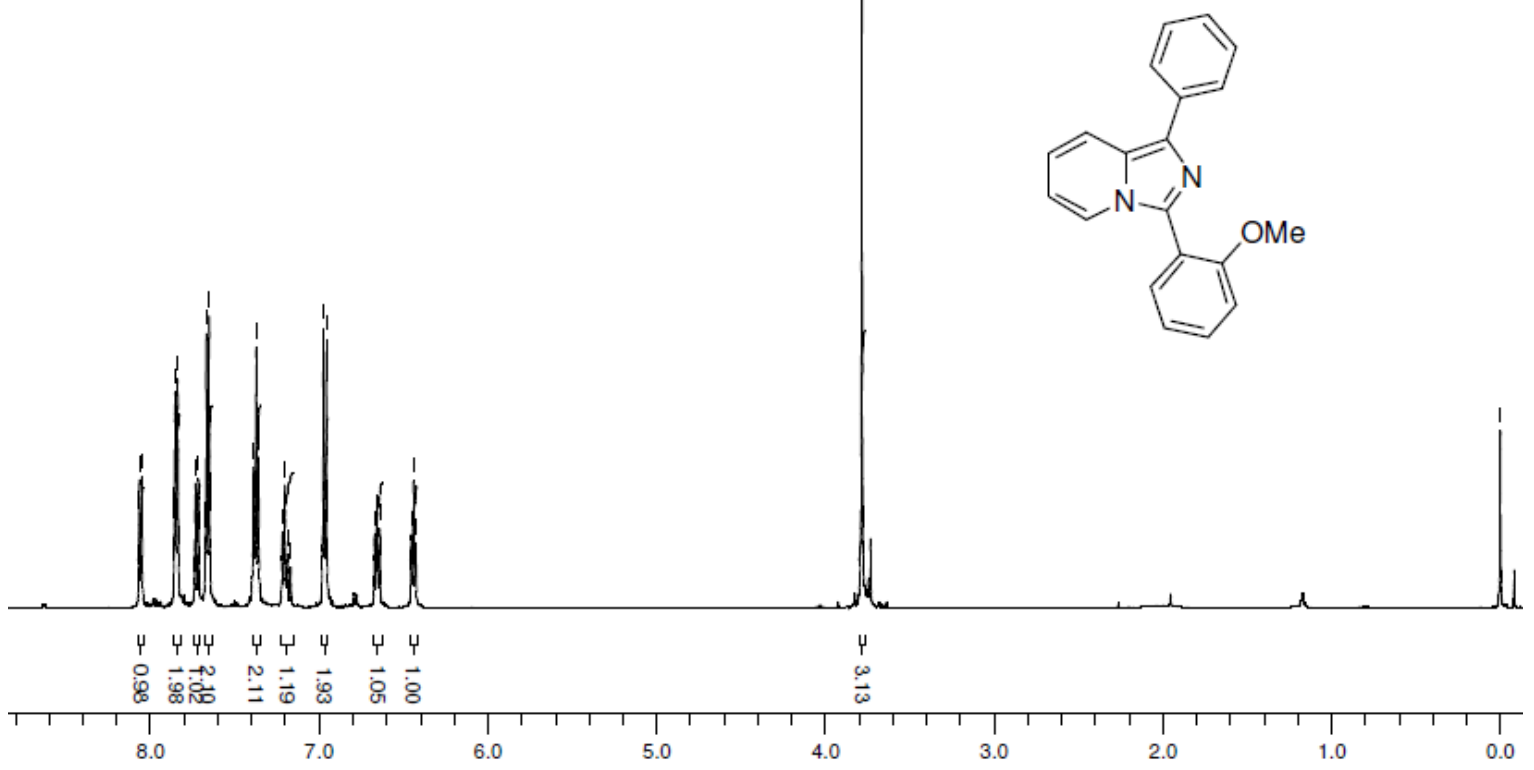

${ }^{1} \mathrm{H}$ NMR of $\mathbf{3 h}$
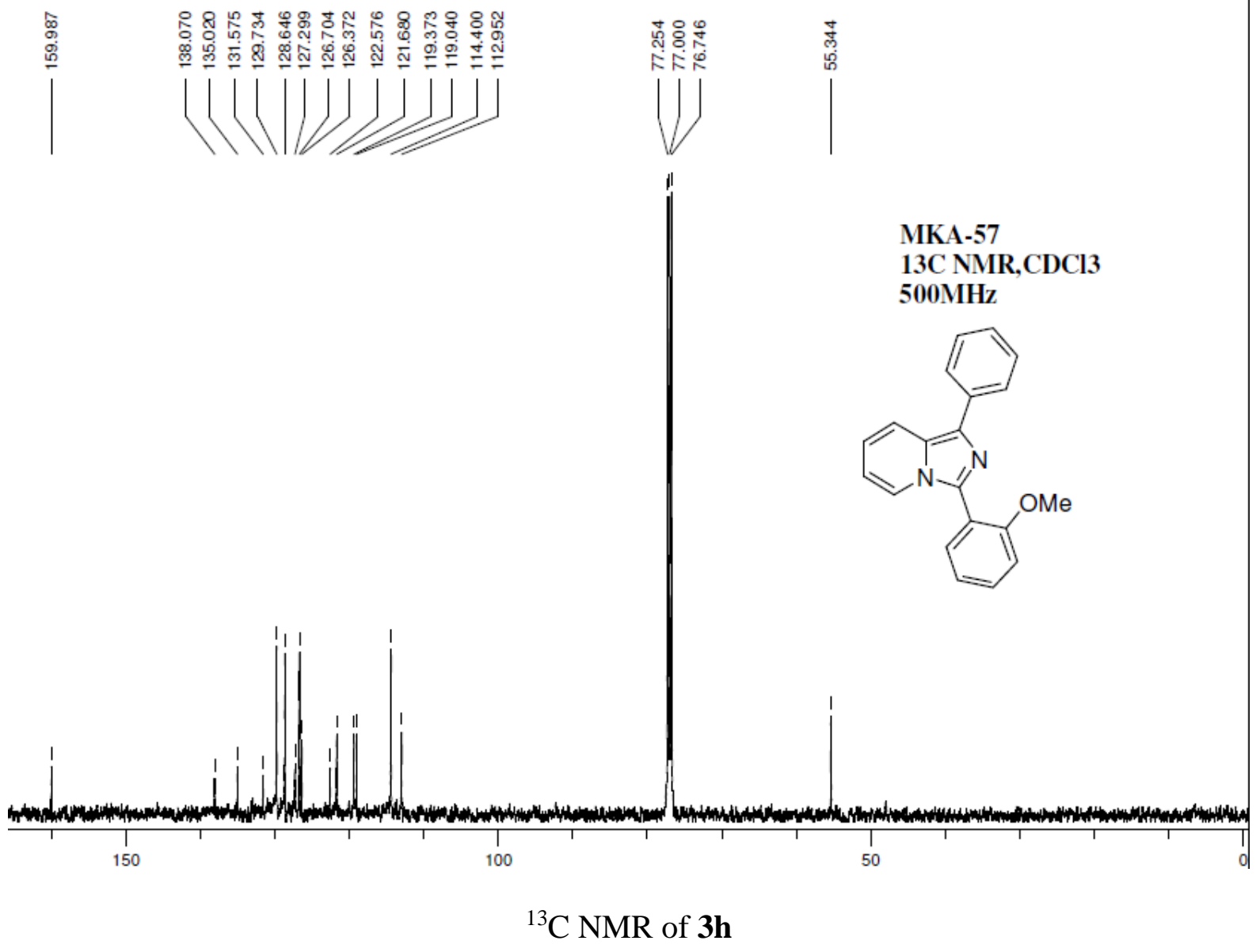


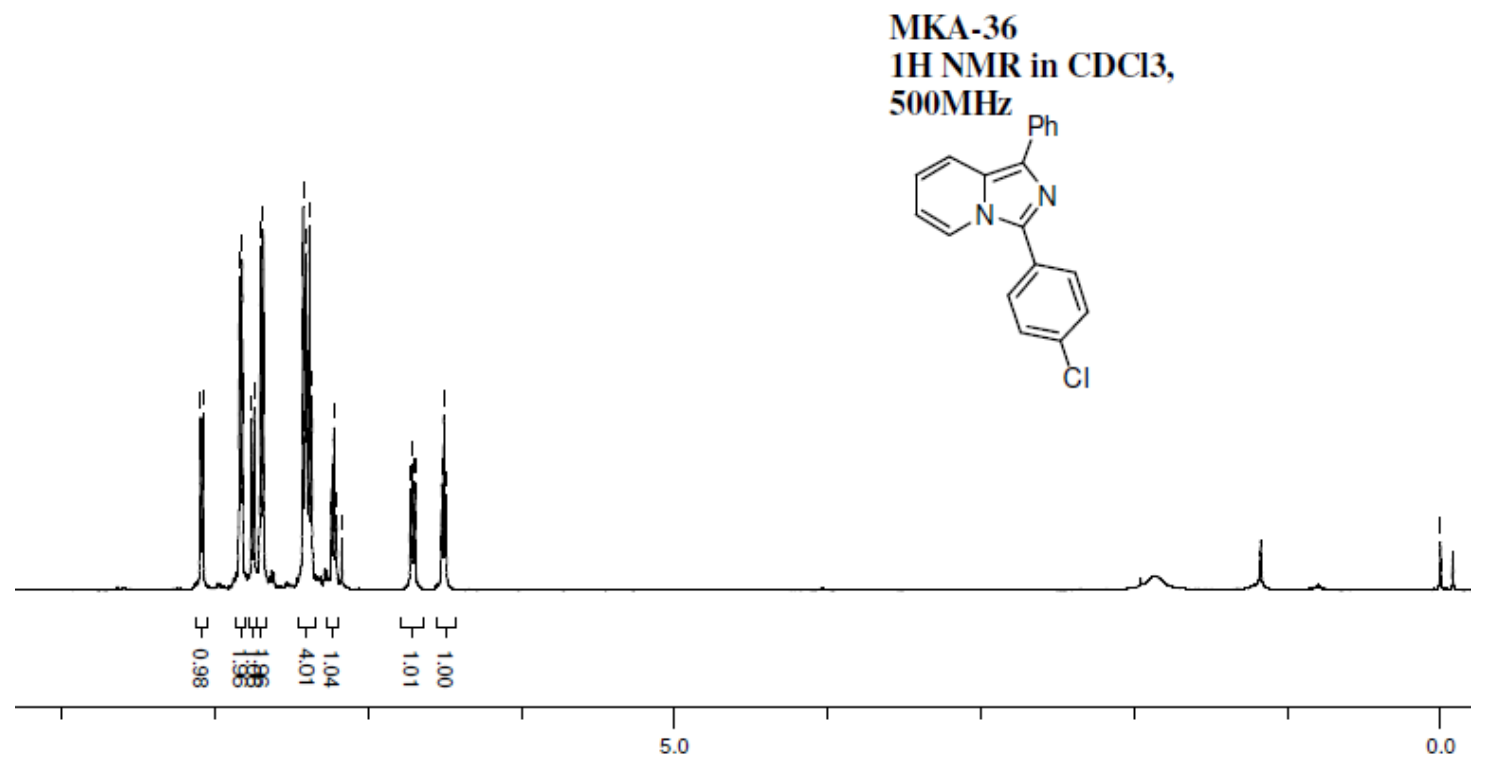

${ }^{1} \mathrm{H}$ NMR of $\mathbf{3 i}$

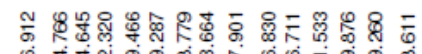

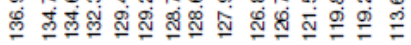

$\lfloor\lfloor\rfloor\rfloor\rfloor\rfloor\rfloor$

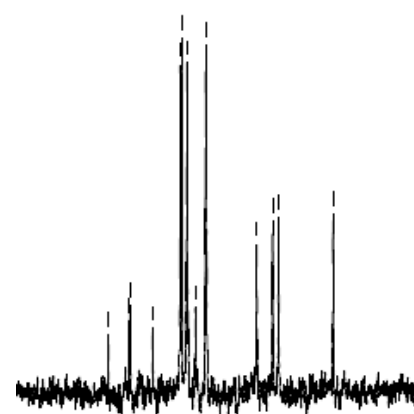

MKA-36

13C NMR in $\mathrm{CDCl} 3$,

$500 \mathrm{MHz}$

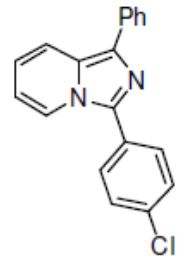

100

50

${ }^{13} \mathrm{C}$ NMR of $\mathbf{3 i}$ 


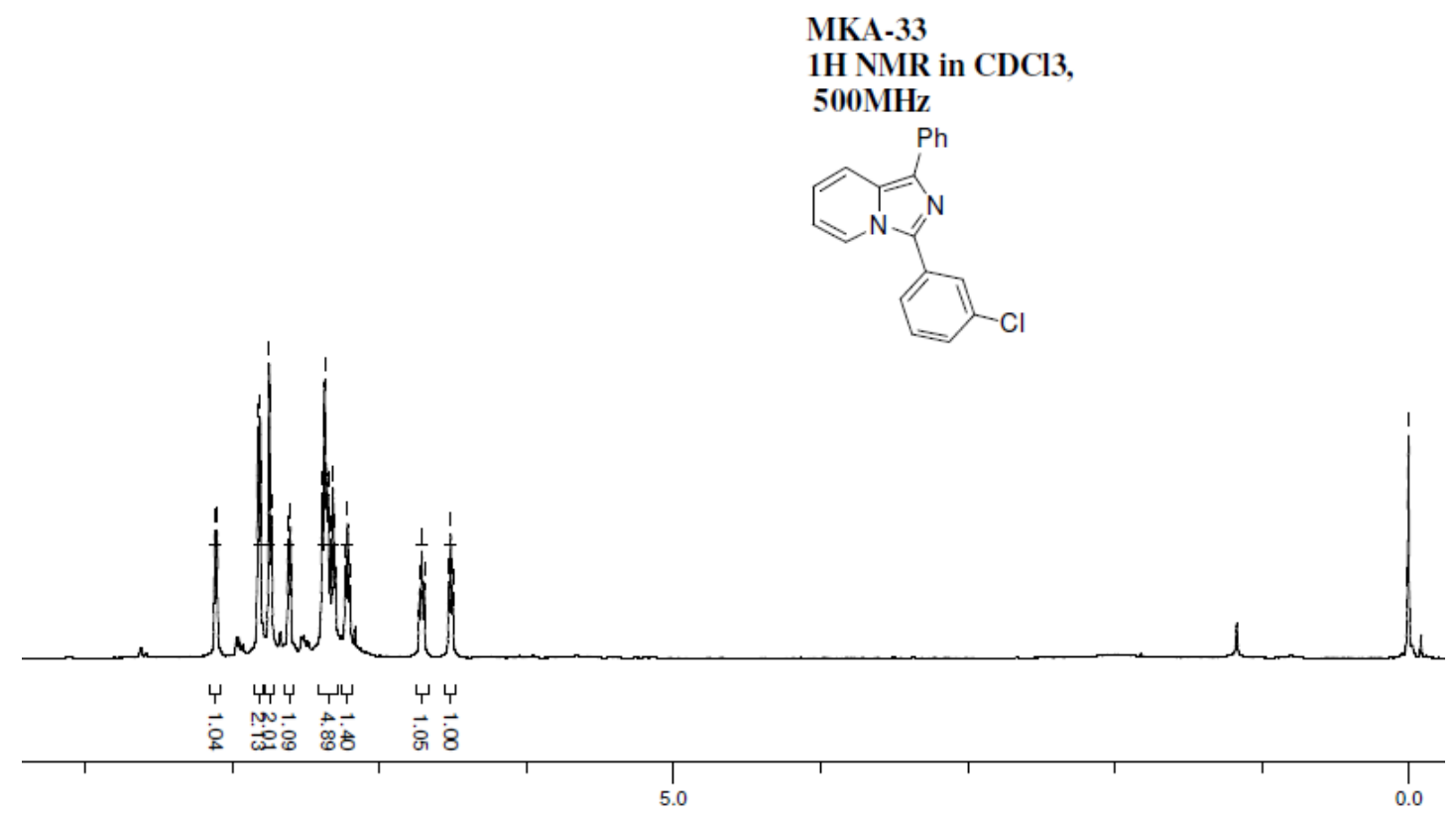

${ }^{1} \mathrm{H}$ NMR of $\mathbf{3 j}$
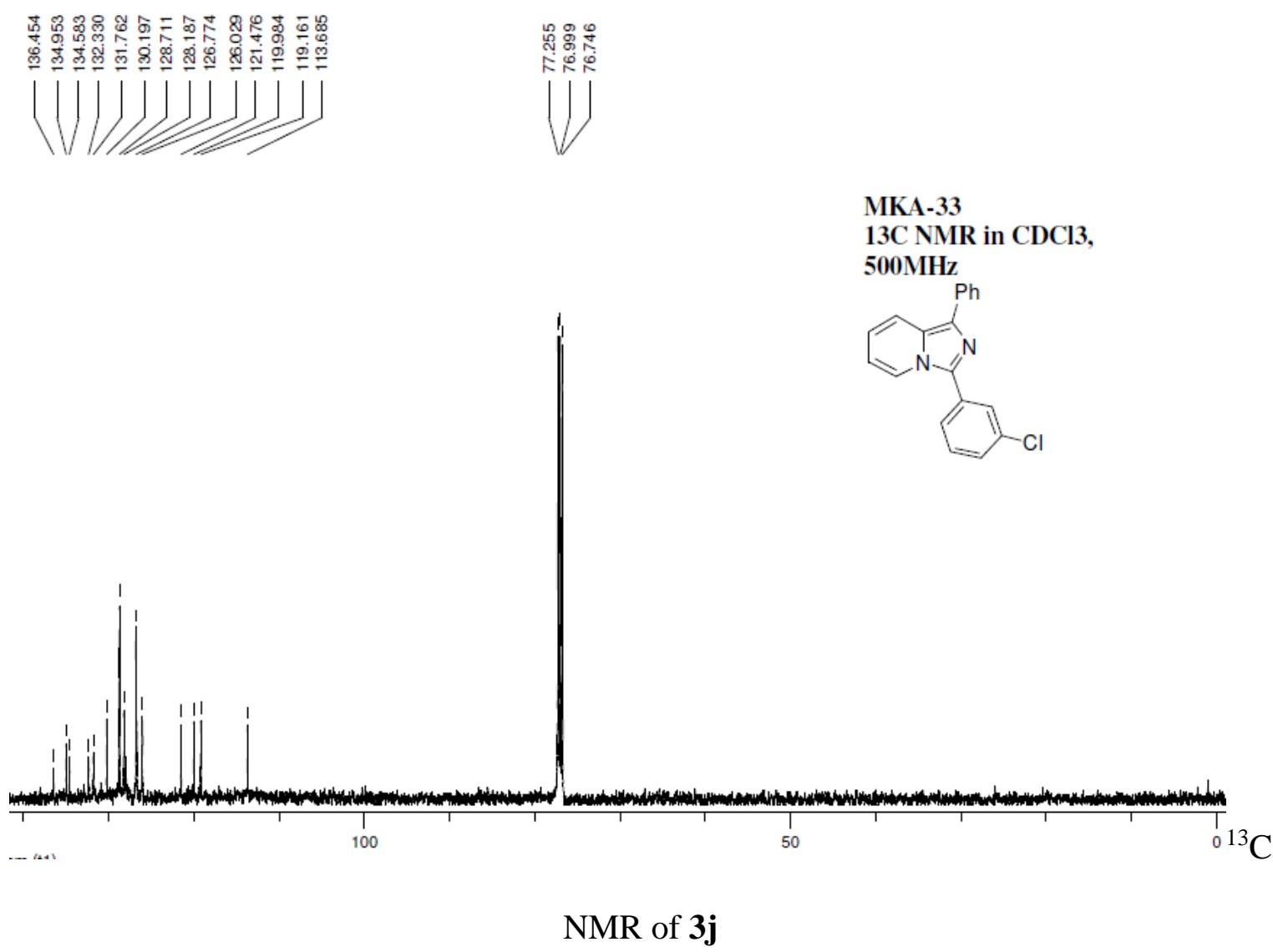


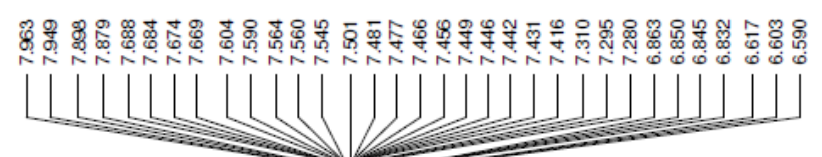

MKA-32

$1 \mathrm{H} \mathrm{NMR}$ in CDCl3,

$500 \mathrm{MHz}$
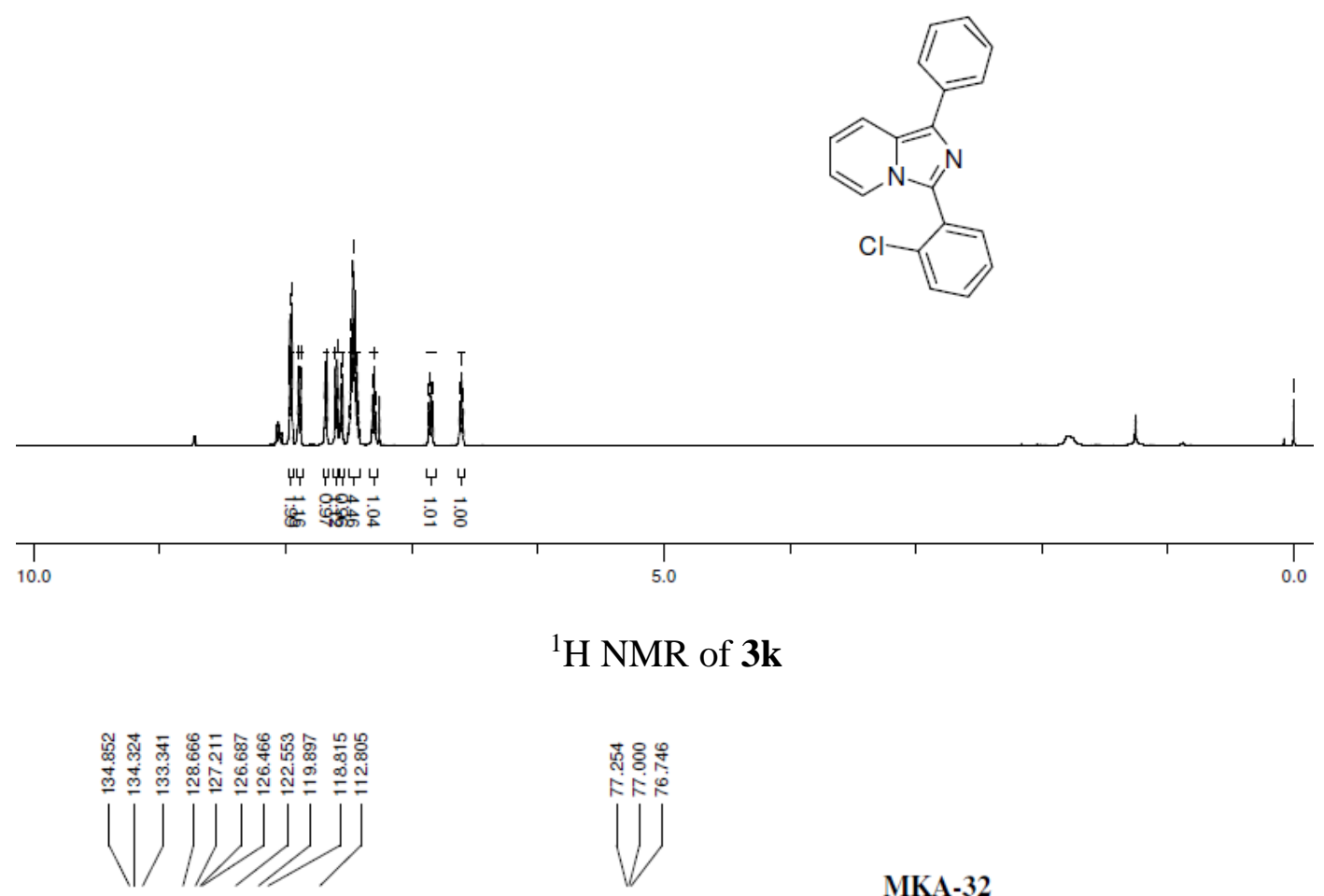

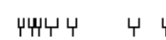

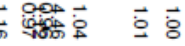

${ }^{1} \mathrm{H}$ NMR of $\mathbf{3 k}$

萬每是

NR。

|V

MKA-32

13C NMR in $\mathrm{CDCl} 3$,

$500 \mathrm{MHz}$
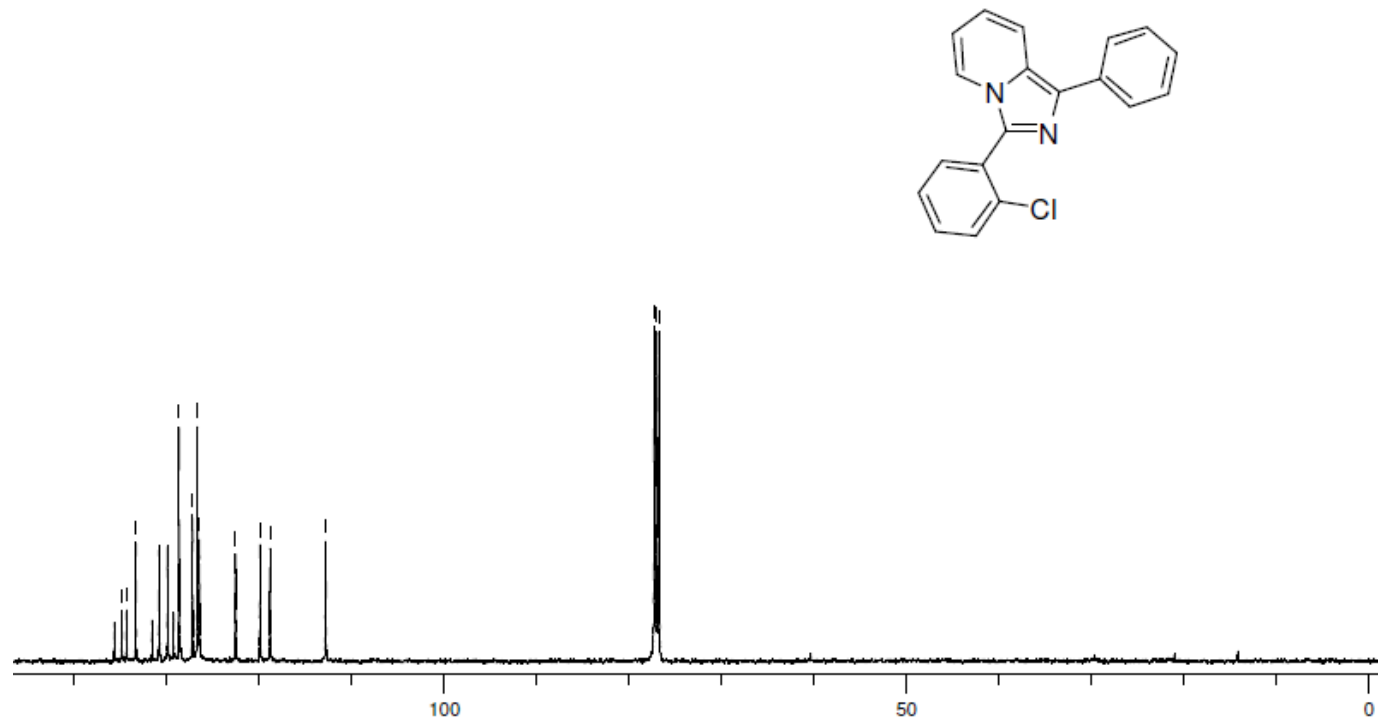

${ }^{13} \mathrm{C}$ NMR of $\mathbf{3 k}$ 
MKA-38

1H NMR in CDCl3,

$500 \mathrm{MHz}$

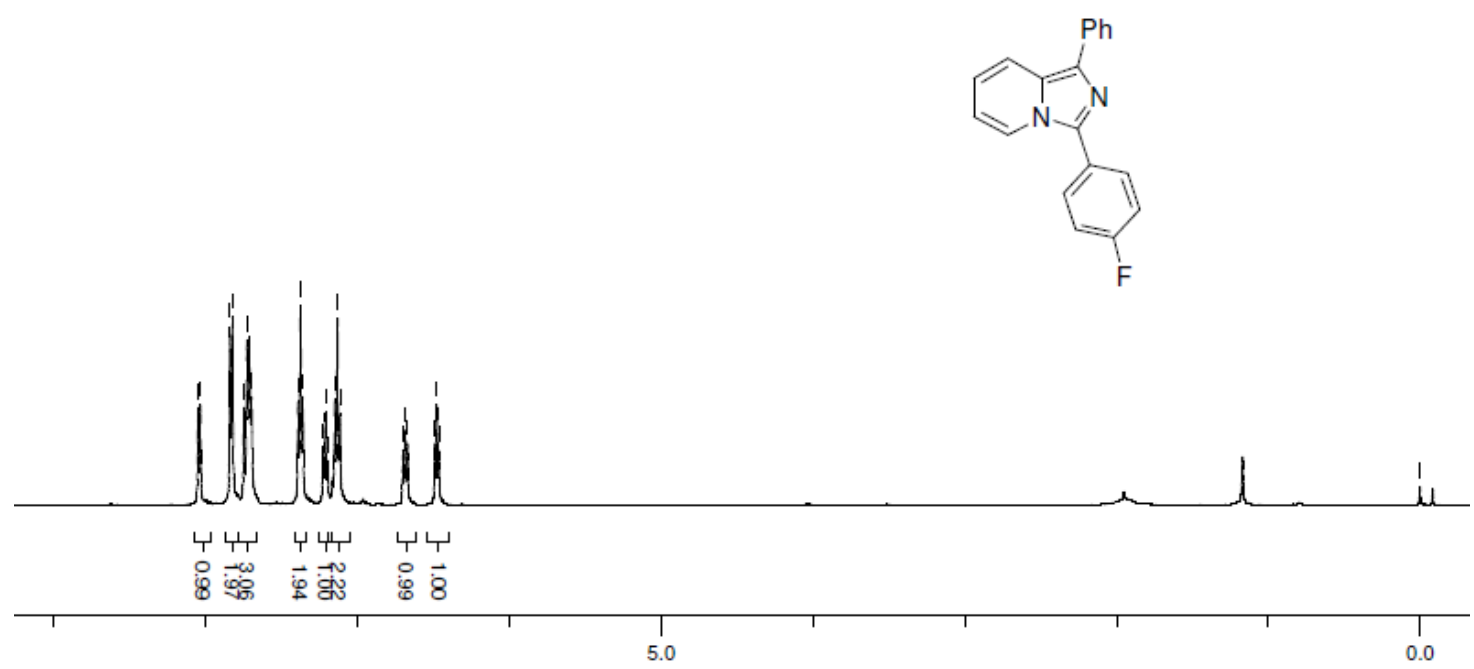

1H NMR of 31

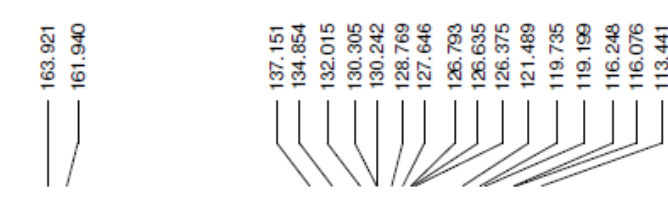

MKA-38

13C NMR in $\mathrm{CDCl} 3$, $500 \mathrm{MHz}$

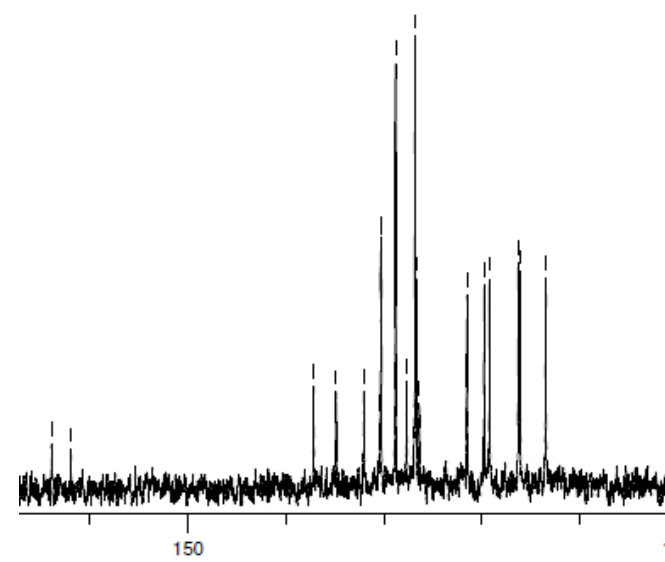

100

${ }^{13} \mathrm{C}$ NMR of 31 


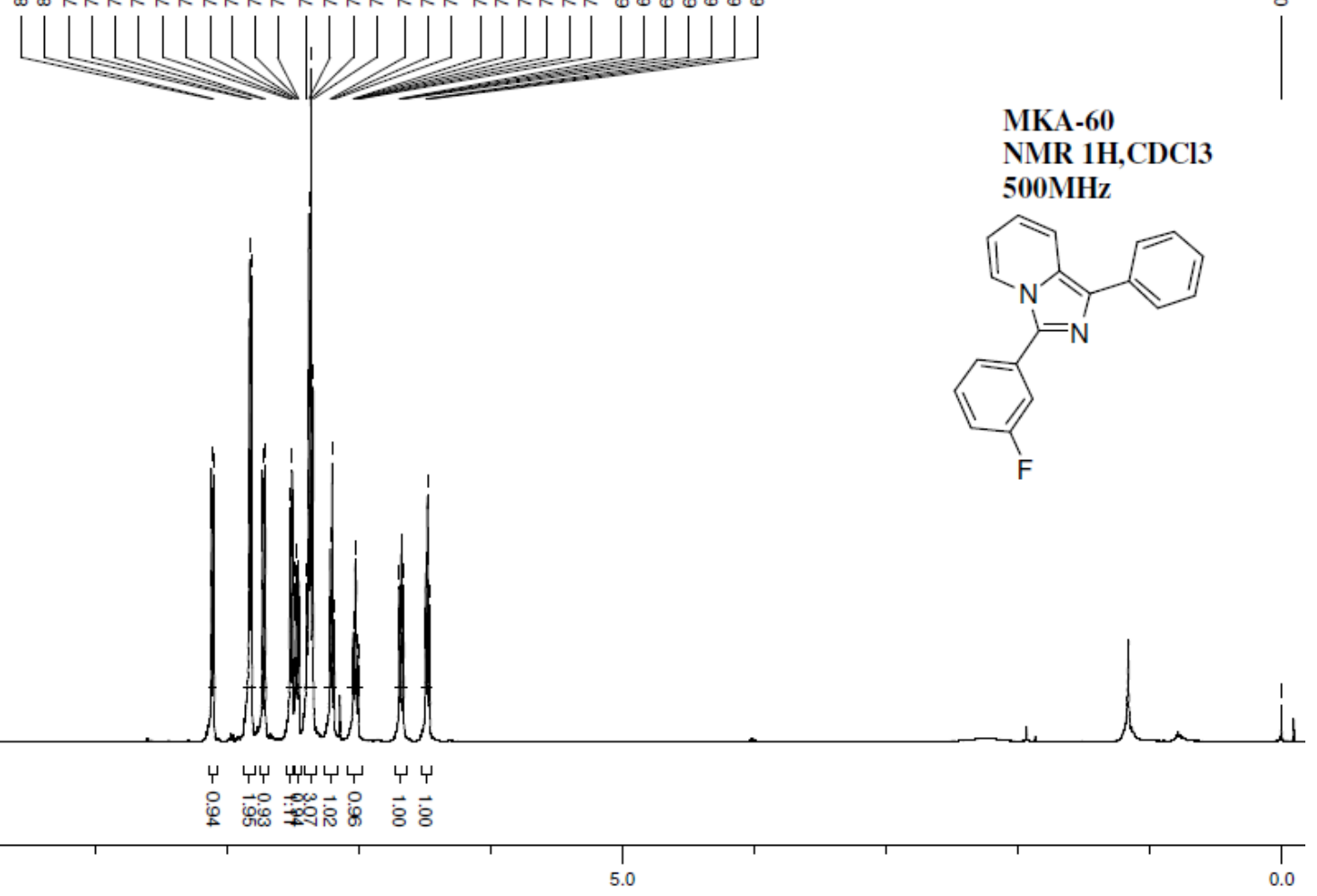

${ }^{1} \mathrm{H}$ NMR of $\mathbf{3 m}$

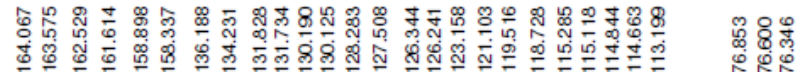
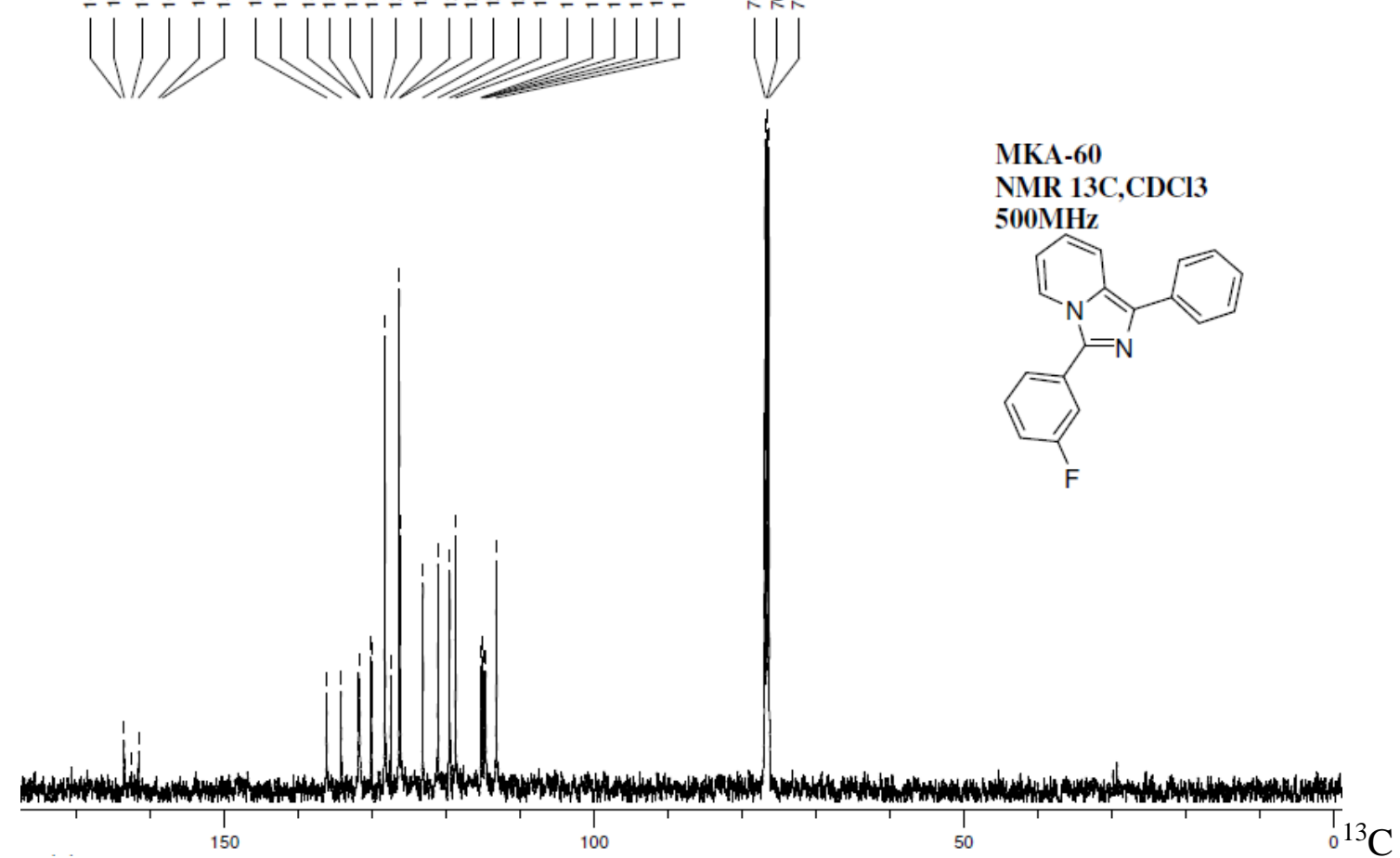

NMR of 3m 


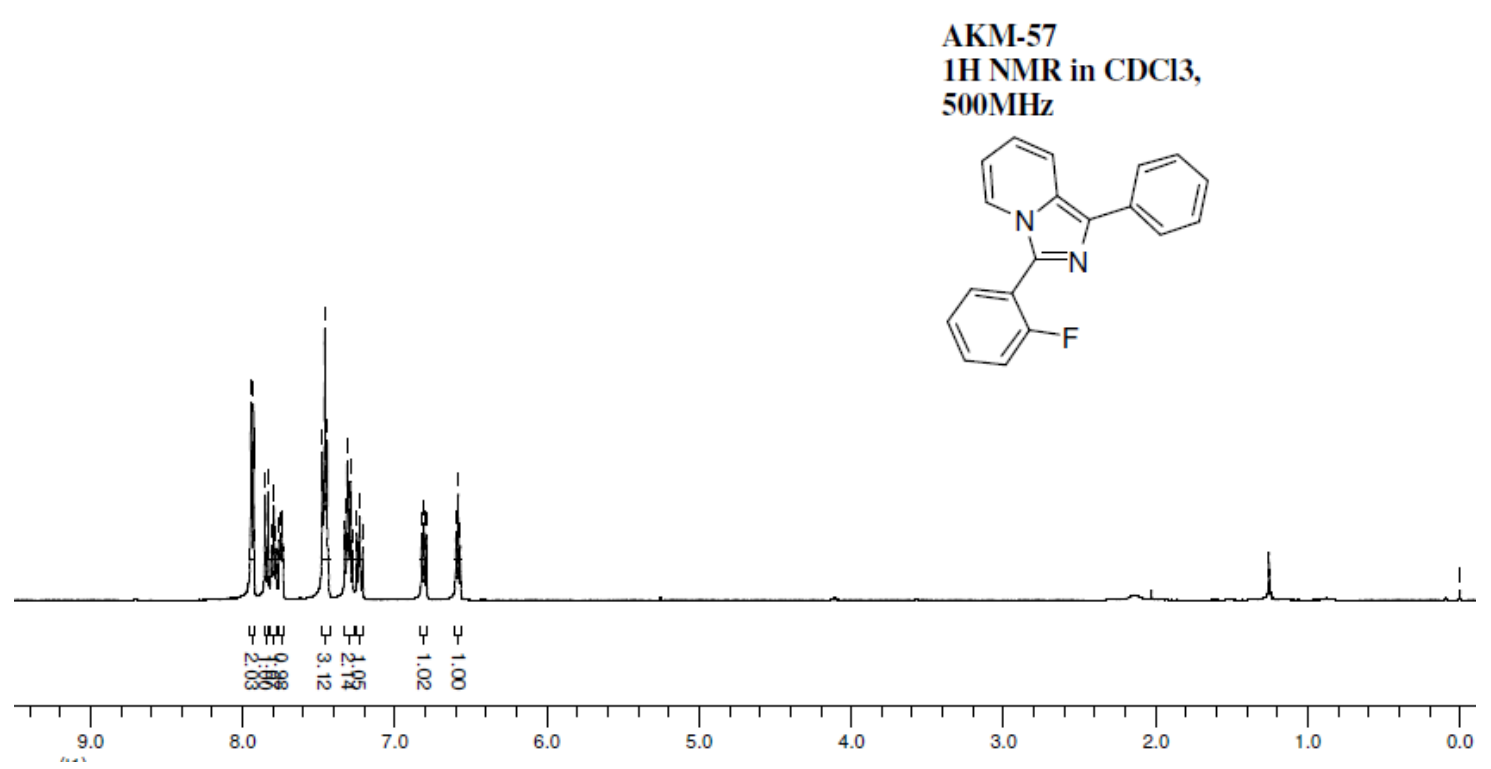

${ }^{1}$ H NMR of 3n

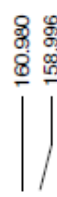

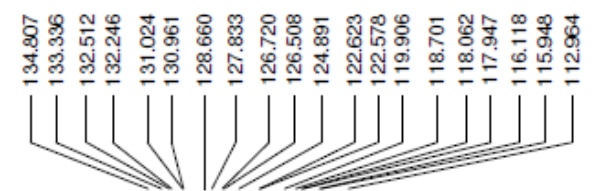

蒙客兑

1)

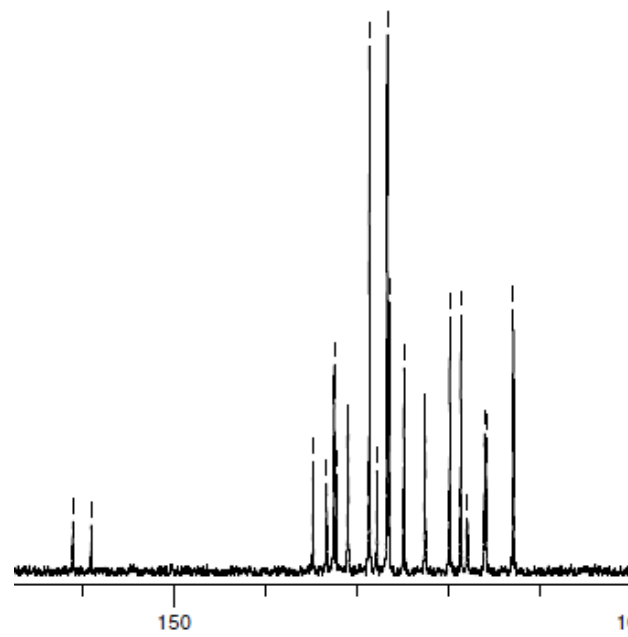

AKM-57

13C NMR in CDCl3, $500 \mathrm{MHz}$

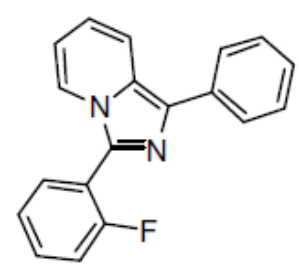

${ }^{3} \mathrm{C}$ NMR of 3 n 


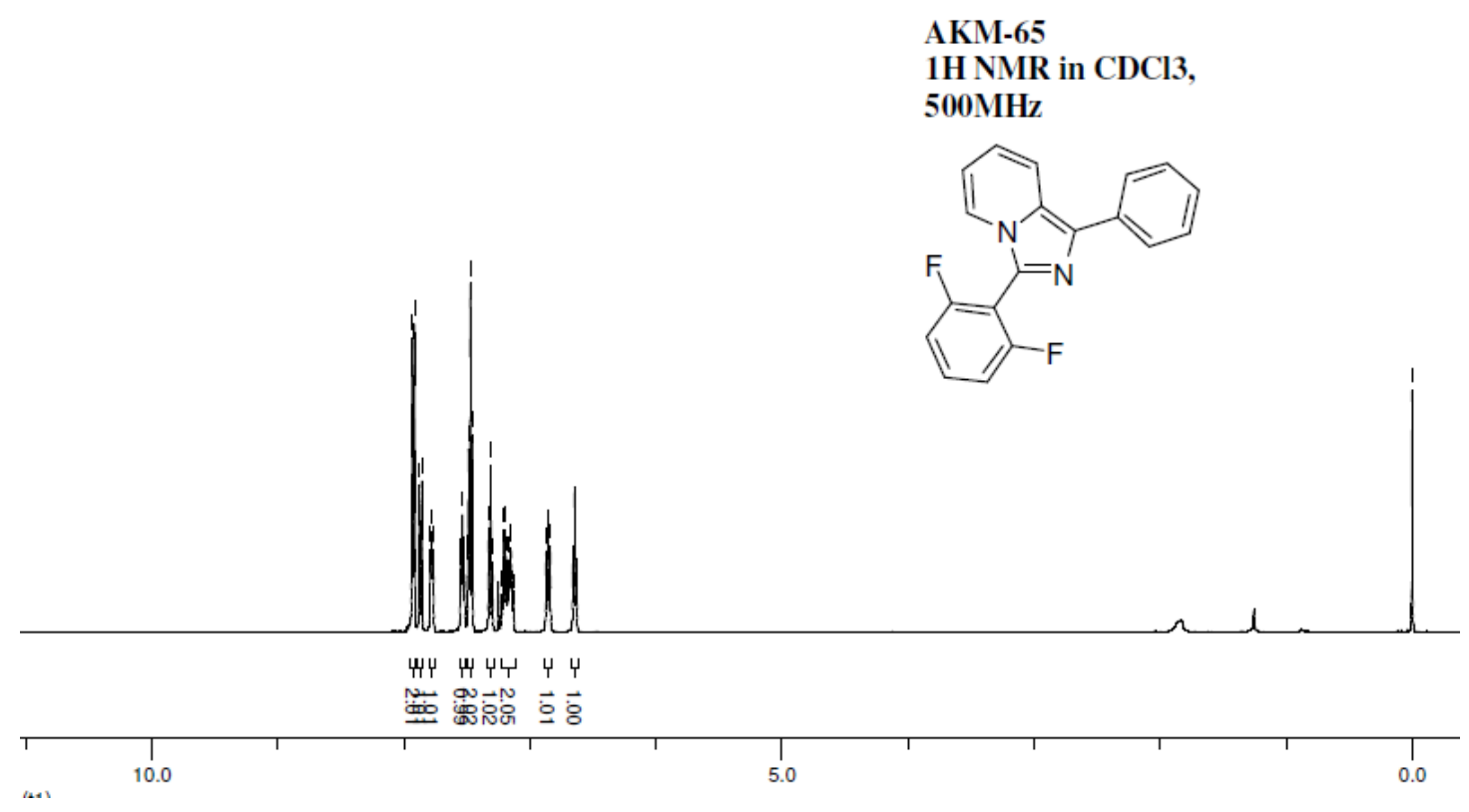

${ }^{1} \mathrm{H}$ NMR of $\mathbf{3 o}$

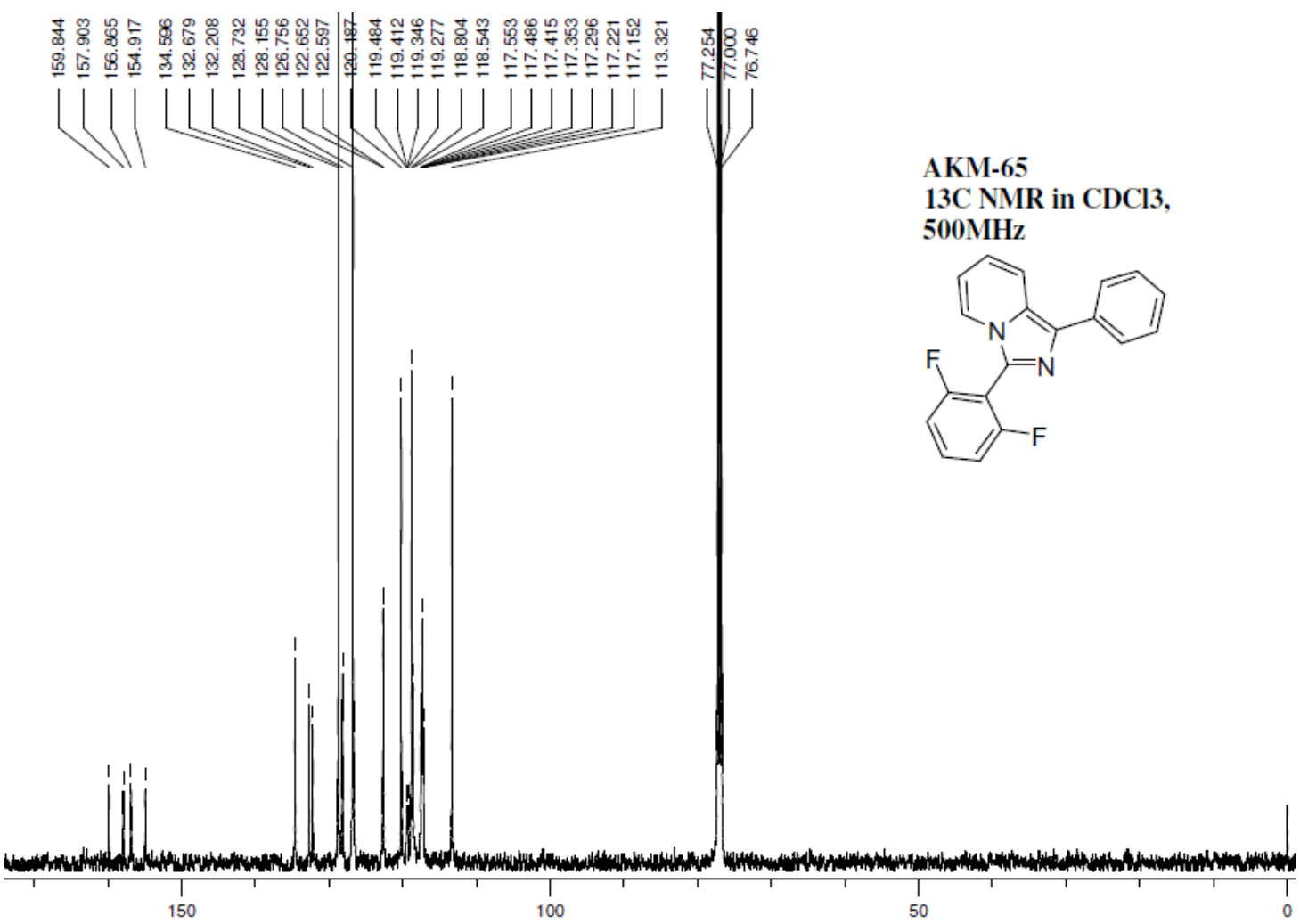

${ }^{13} \mathrm{C}$ NMR of 30 
MKA-37

1H NMR in CDCl3,

$500 \mathrm{MHz}$

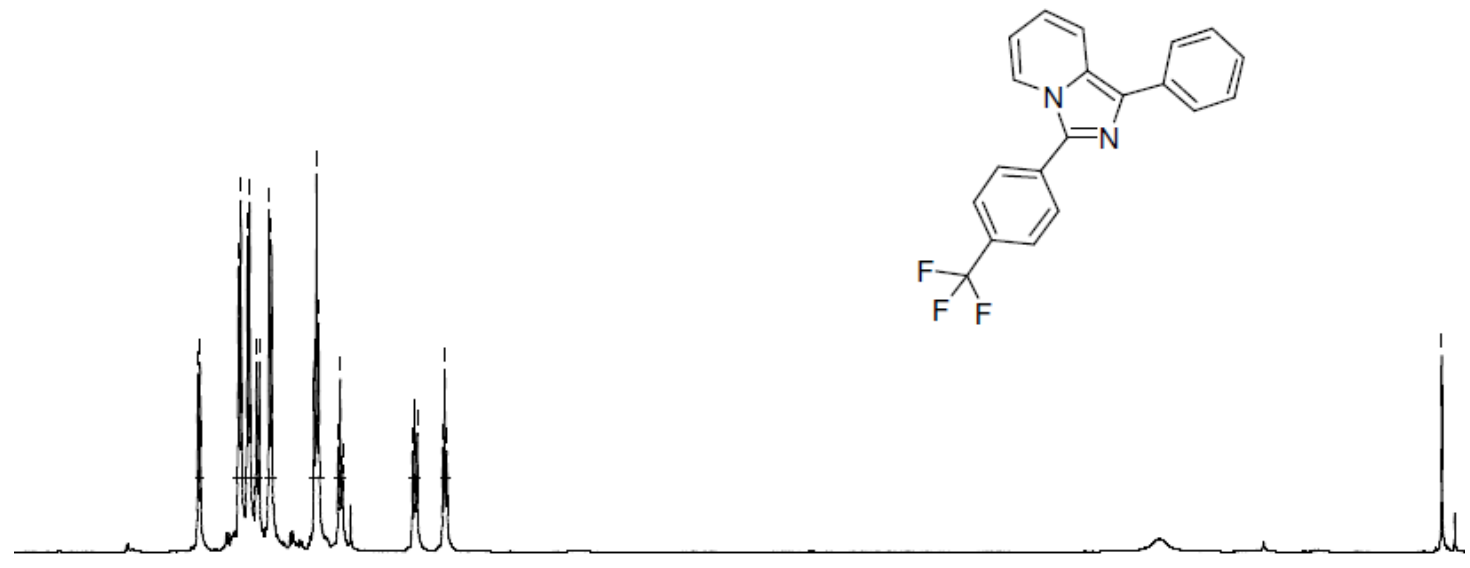

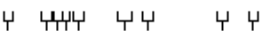

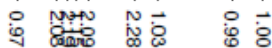

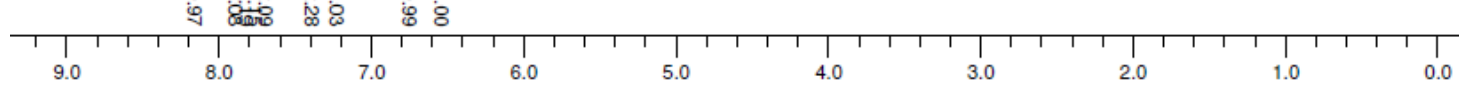

${ }^{1} \mathrm{H}$ NMR of $\mathbf{3 p}$

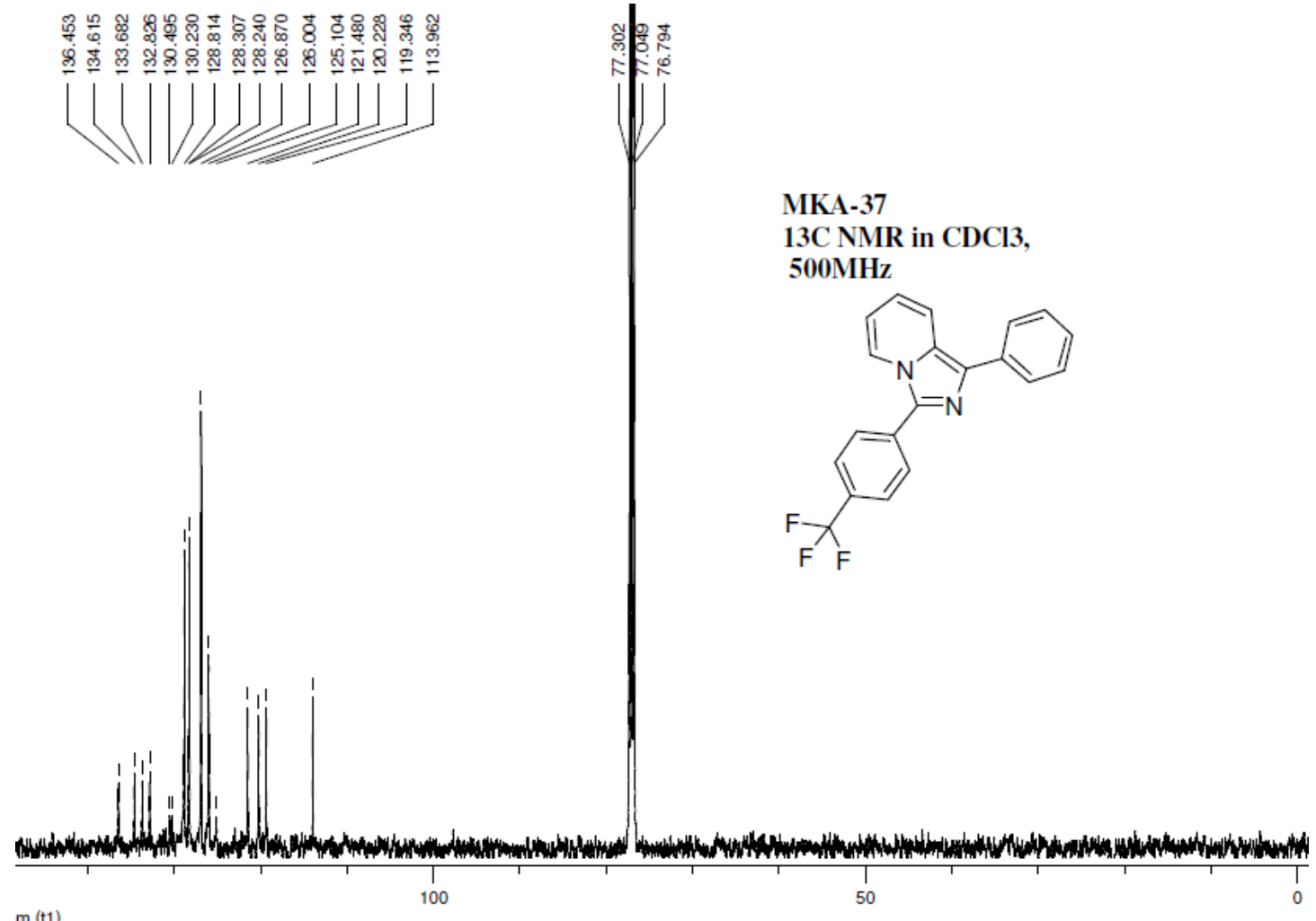

${ }^{13} \mathrm{C}$ NMR of $\mathbf{3 p}$ 
MKA-82

1H NMR in CDCl3,

$500 \mathrm{MHz}$
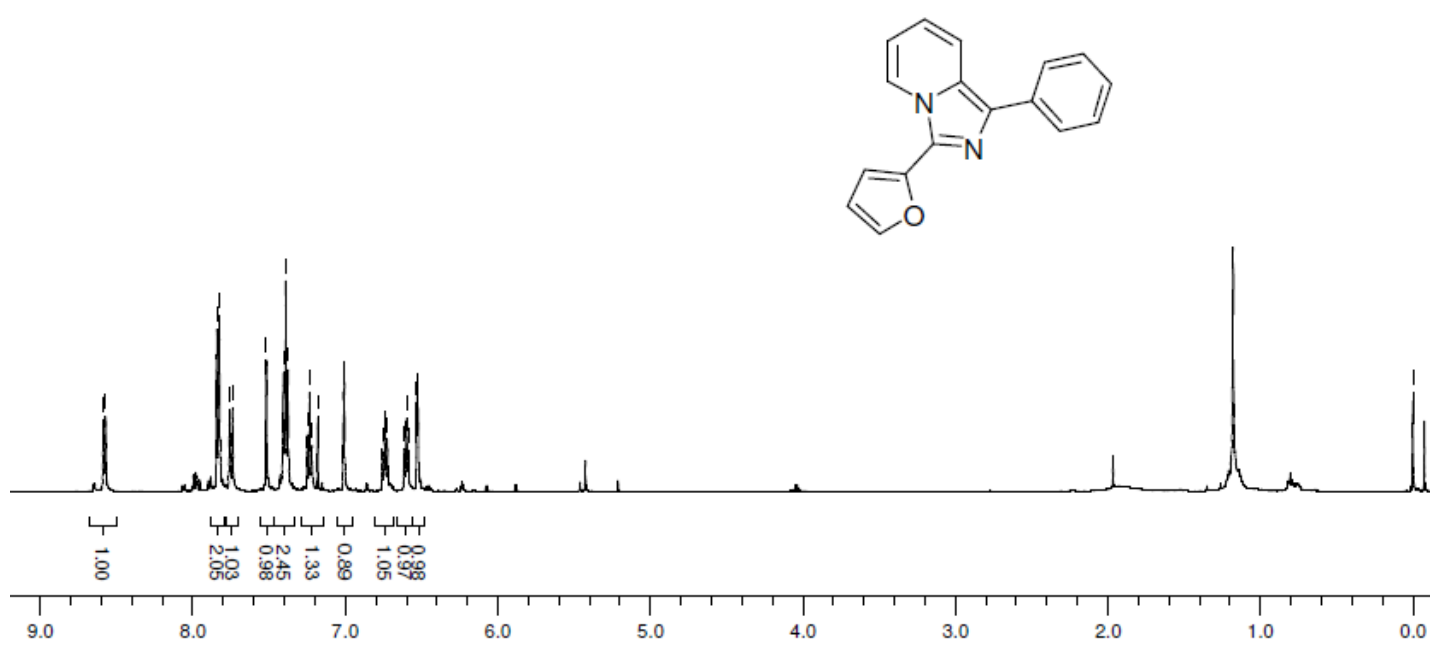

${ }^{1} \mathrm{H}$ NMR of $\mathbf{3 q}$

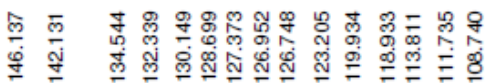
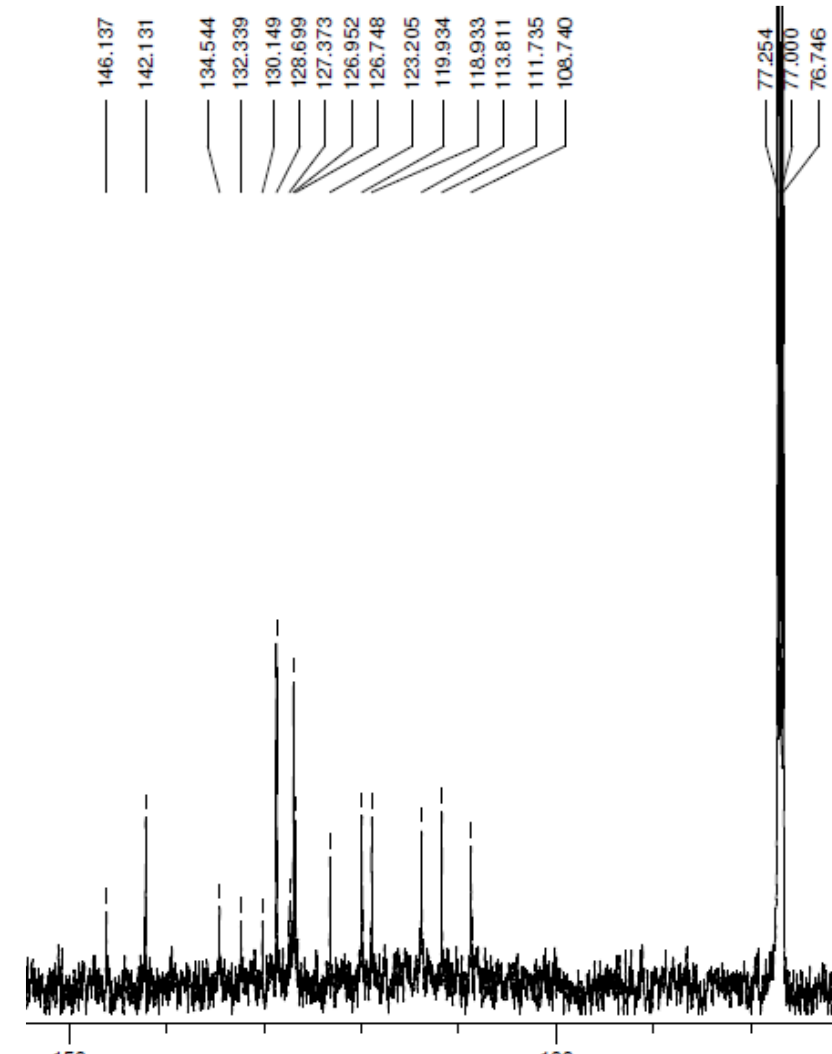

MKA-82

13C NMR in CDCl3,

$500 \mathrm{MHz}$

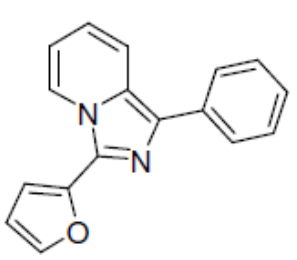

150

100

${ }^{13} \mathrm{C}$ NMR of $\mathbf{3 q}$ 


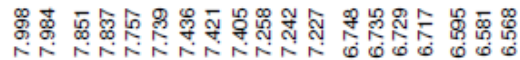

MKA-62

$1 \mathrm{H}$ NMR in $\mathrm{CDCl} 3$,

$500 \mathrm{MHz}$

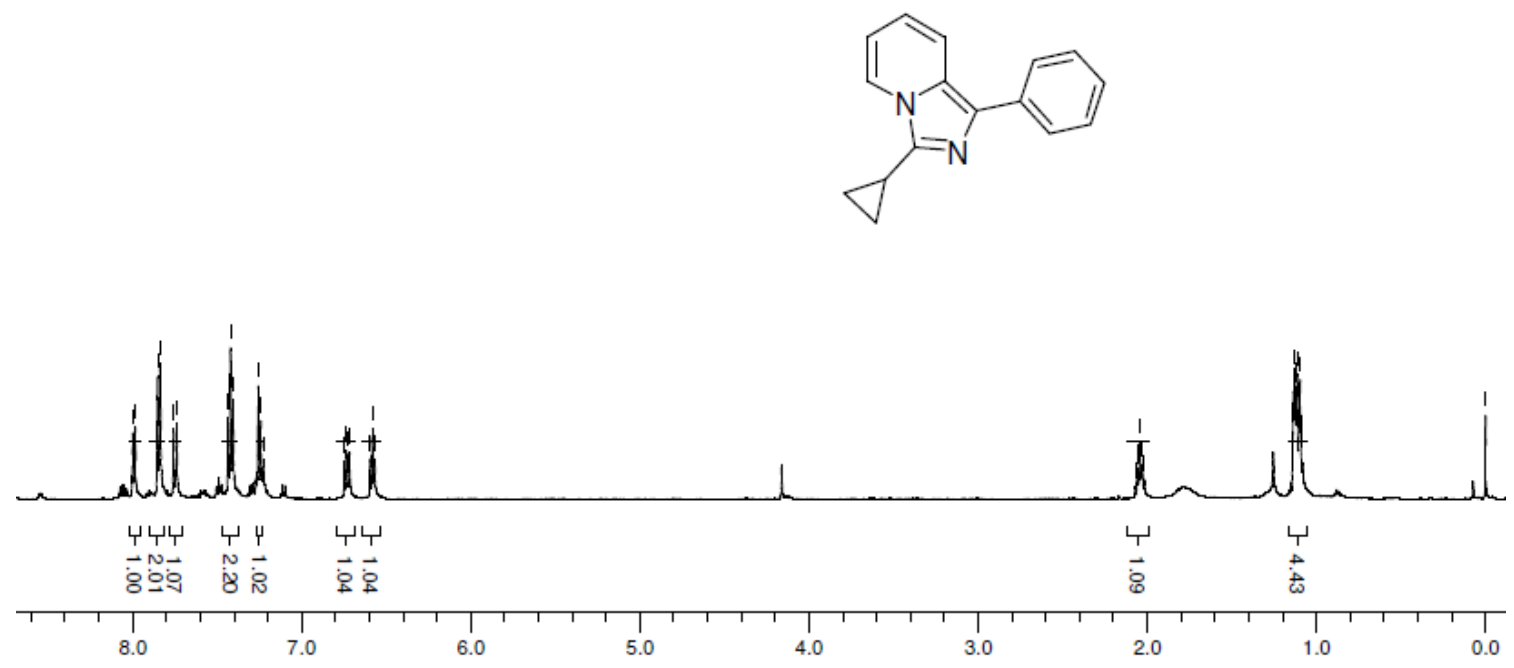

${ }^{1} \mathrm{H}$ NMR of $\mathbf{3 s}$

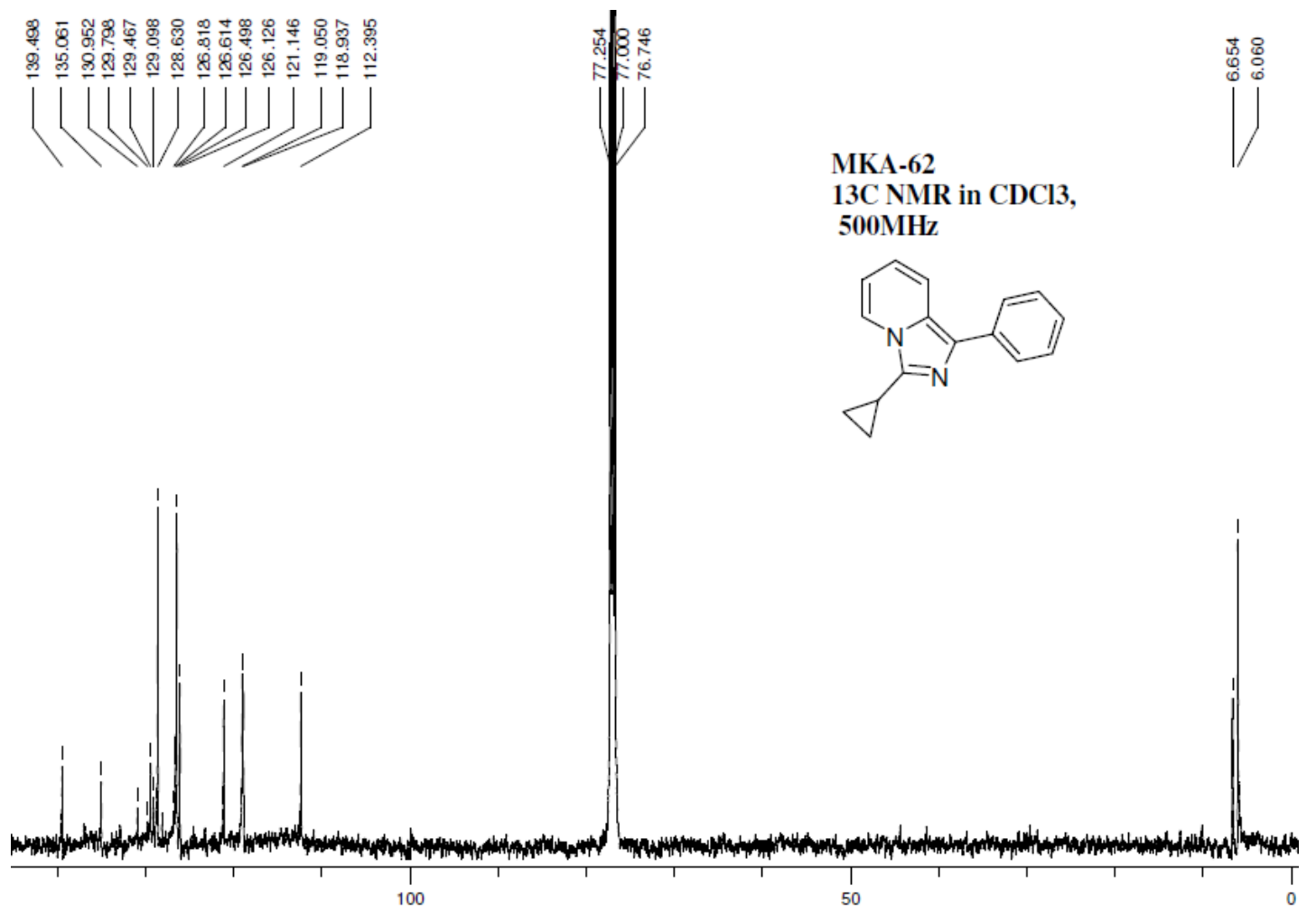

${ }^{13} \mathrm{C}$ NMR of $3 \mathrm{~s}$ 


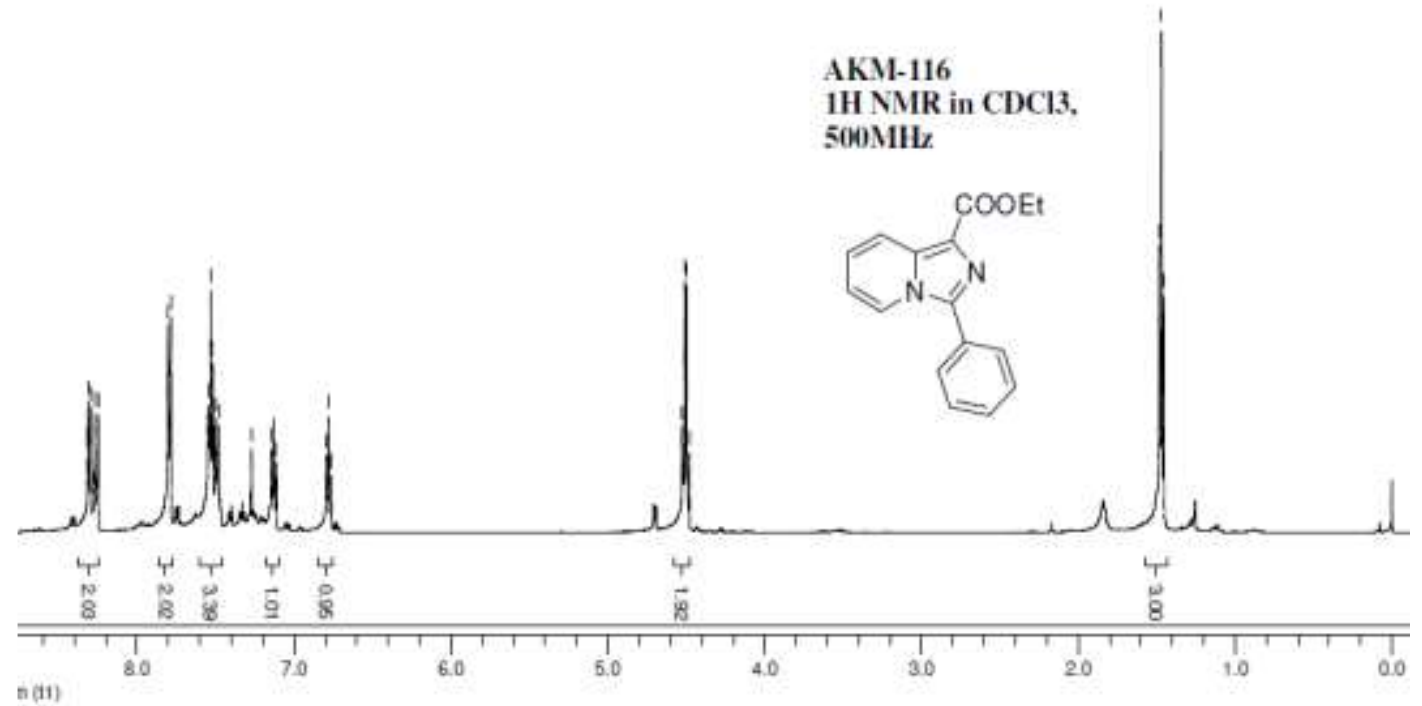

${ }^{1} \mathrm{H}$ NMR of $\mathbf{3 u}$
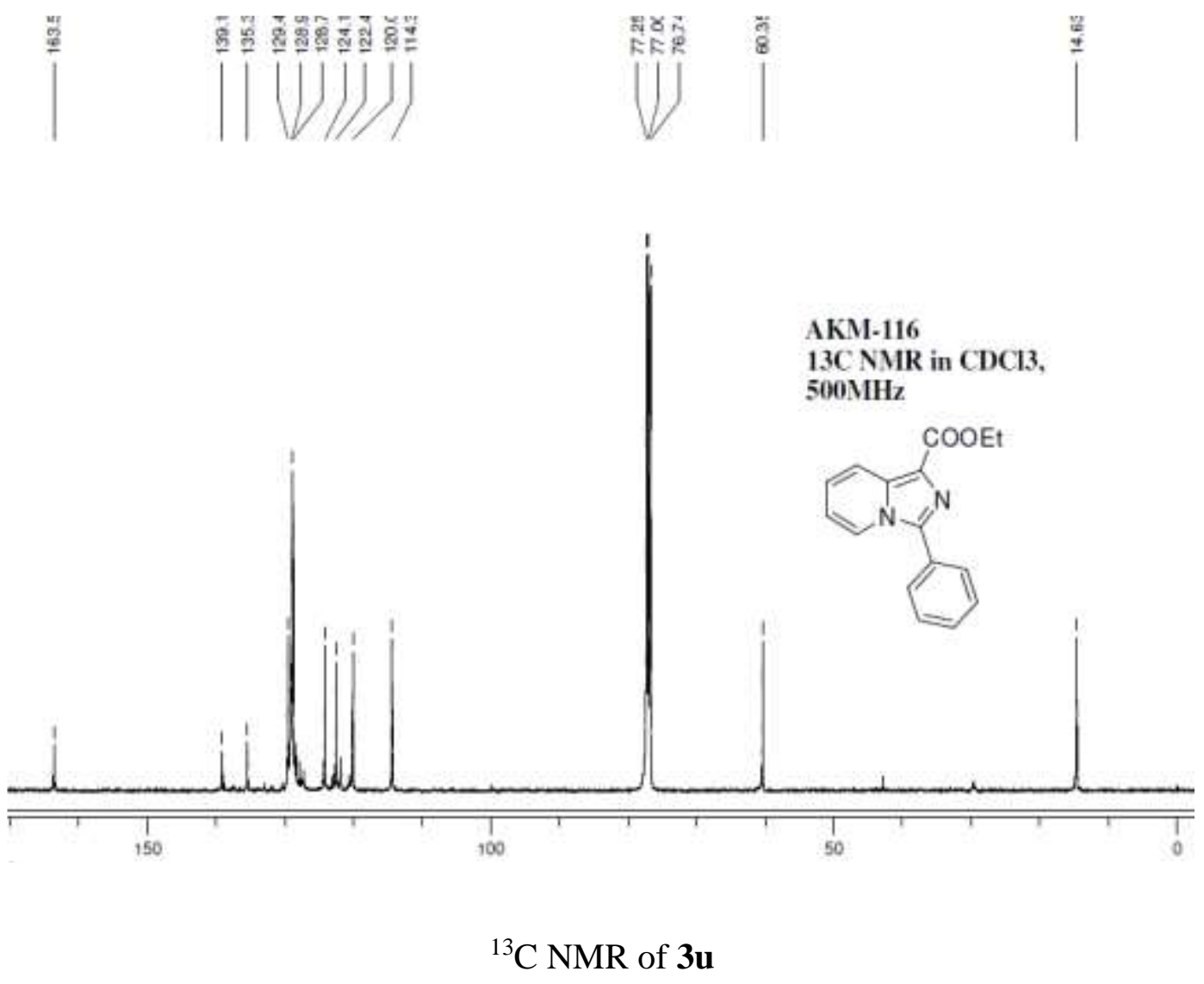


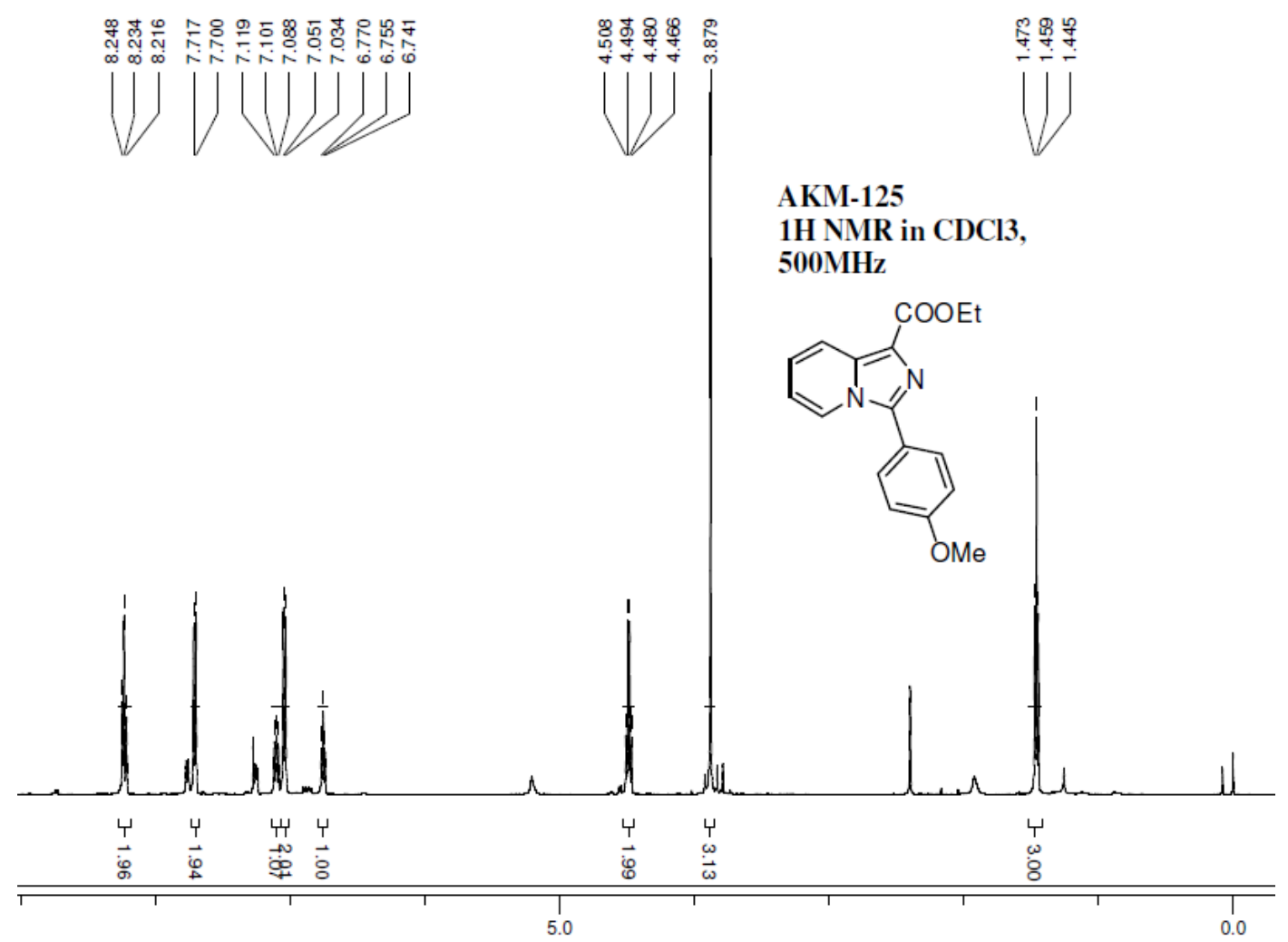

${ }^{1} \mathrm{H}$ NMR of $\mathbf{3 v}$

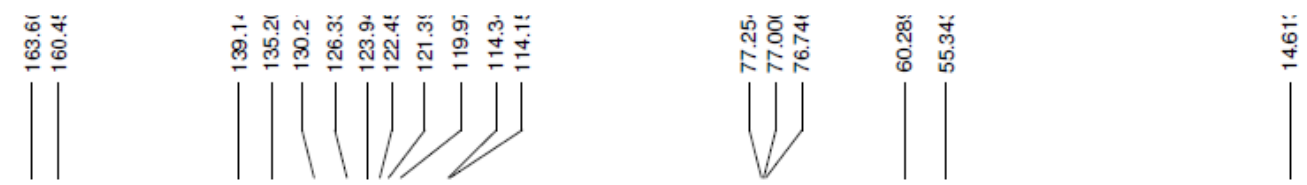

AKM-125

13C NMR in CDCl3,

$500 \mathrm{MHz}$
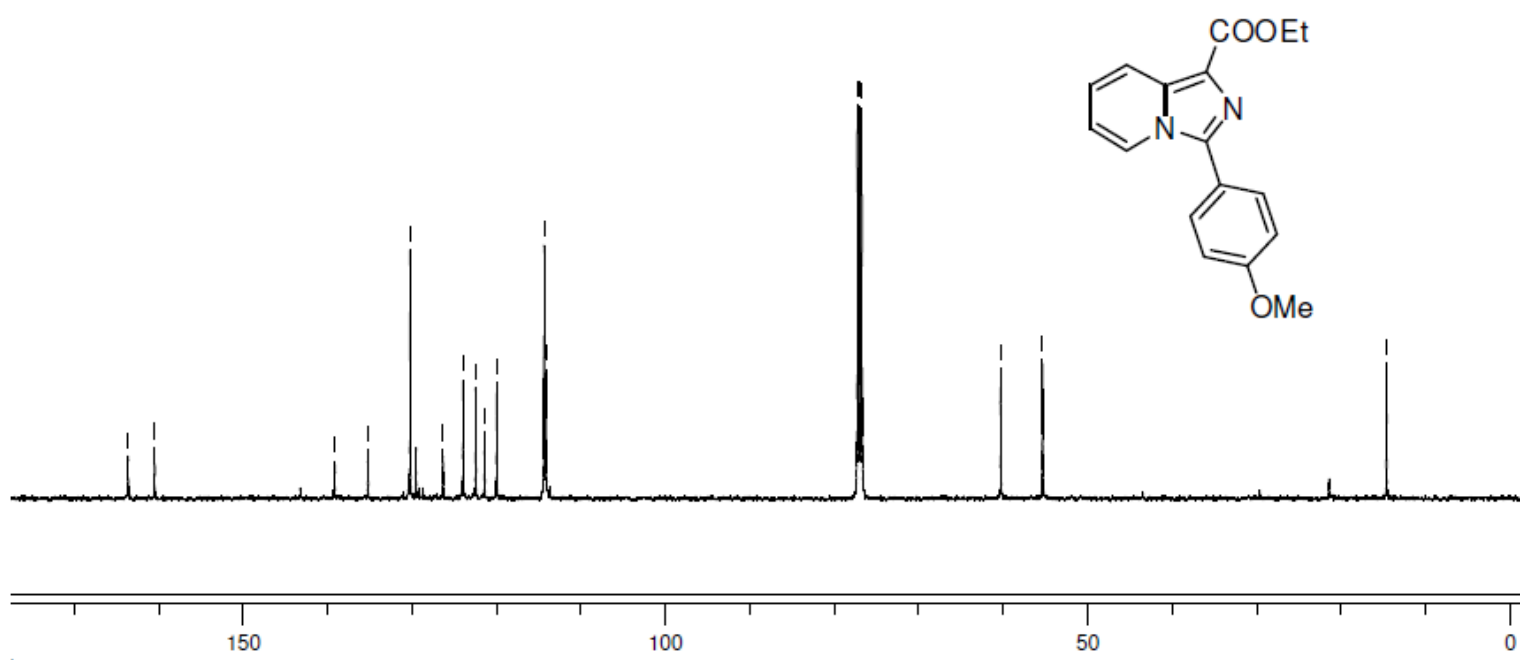

${ }^{13} \mathrm{C}$ NMR of $\mathbf{3 v}$ 


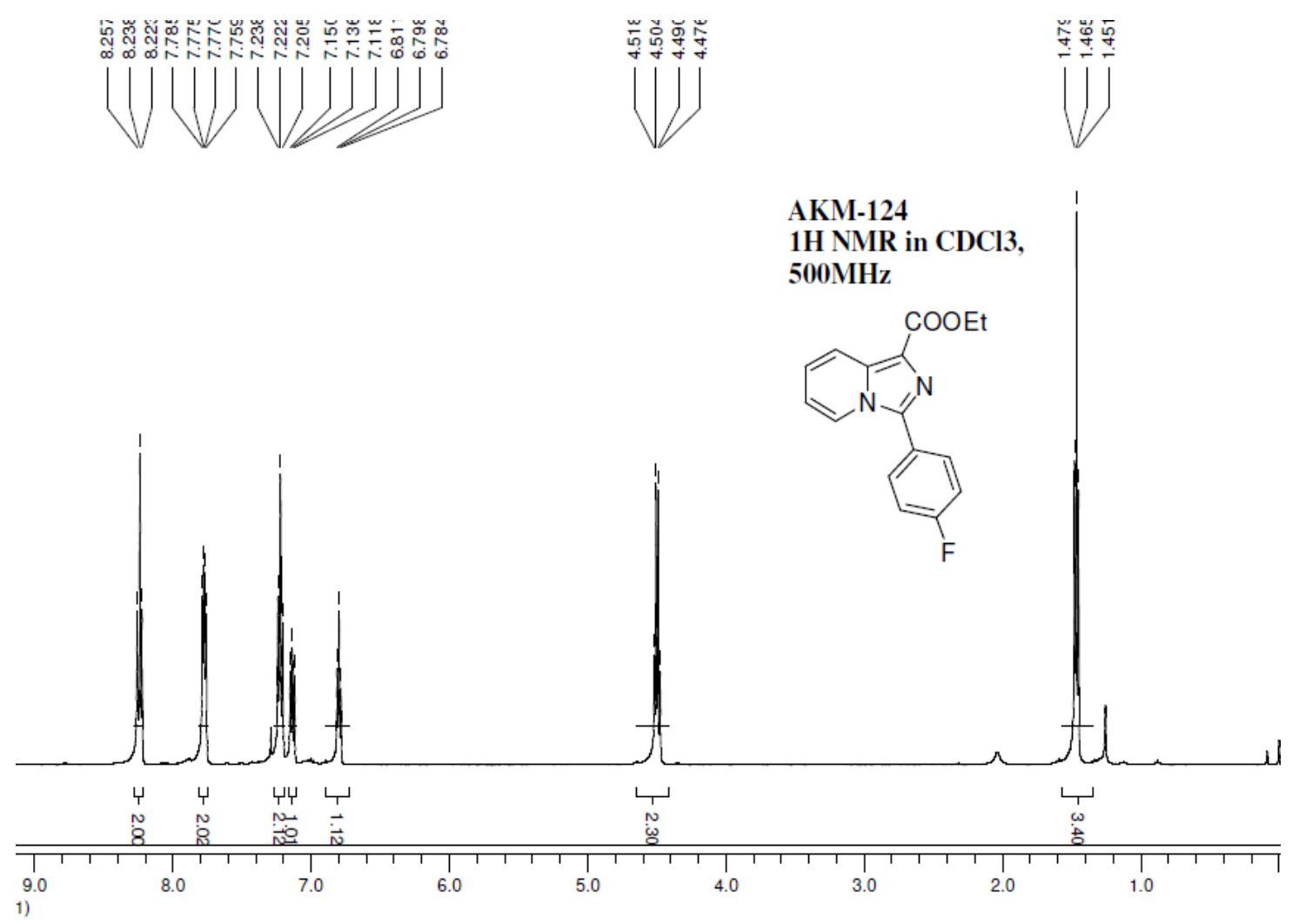

${ }^{1} \mathrm{H}$ NMR of $\mathbf{3 w}$

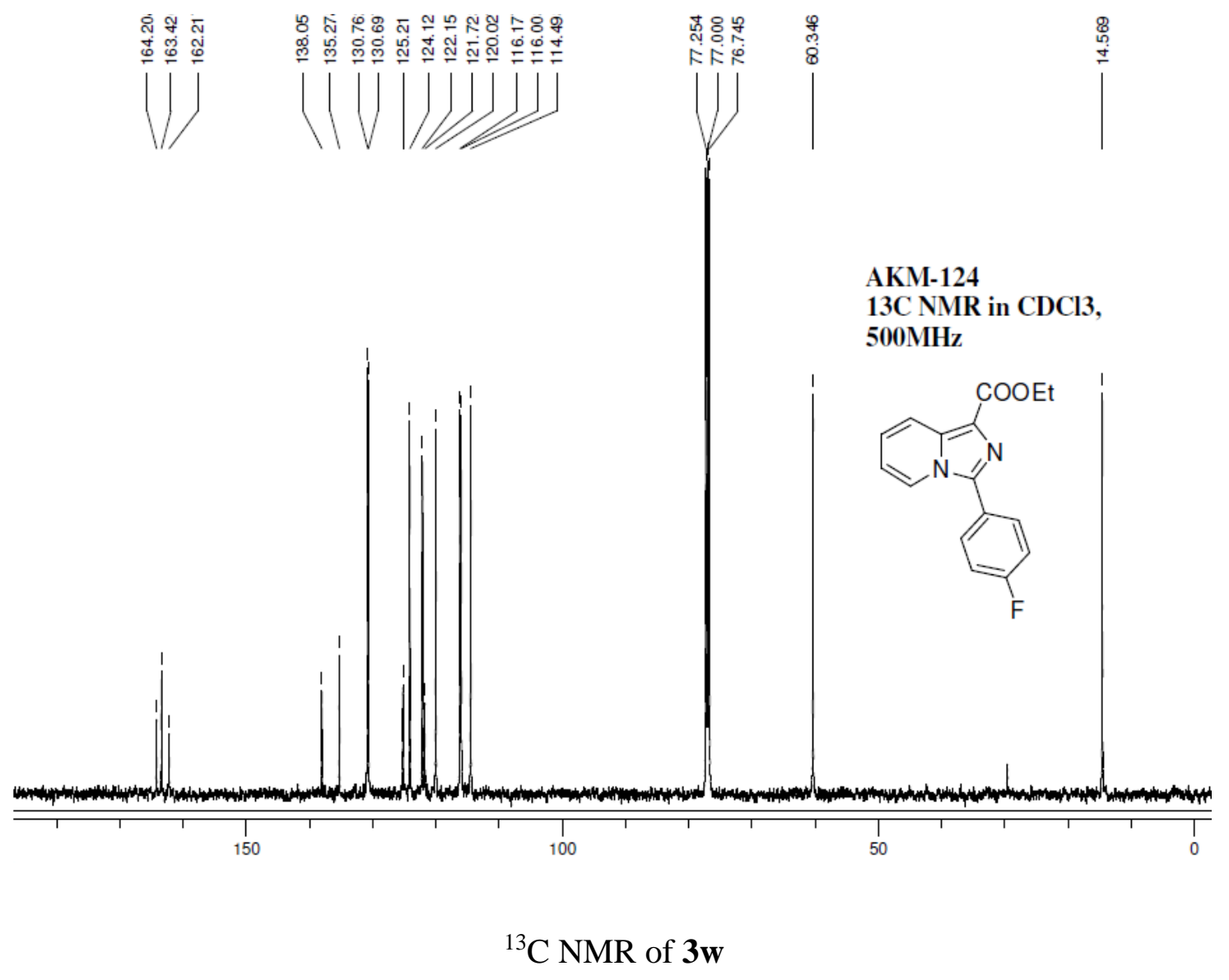




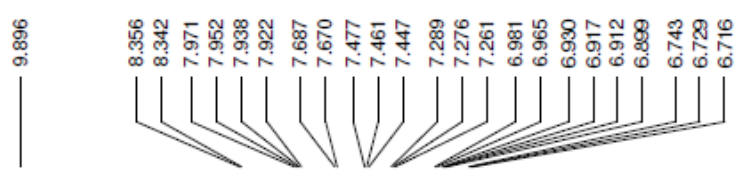

AKM-50

1H NMR in DMSO-d6, $500 \mathrm{MHz}$
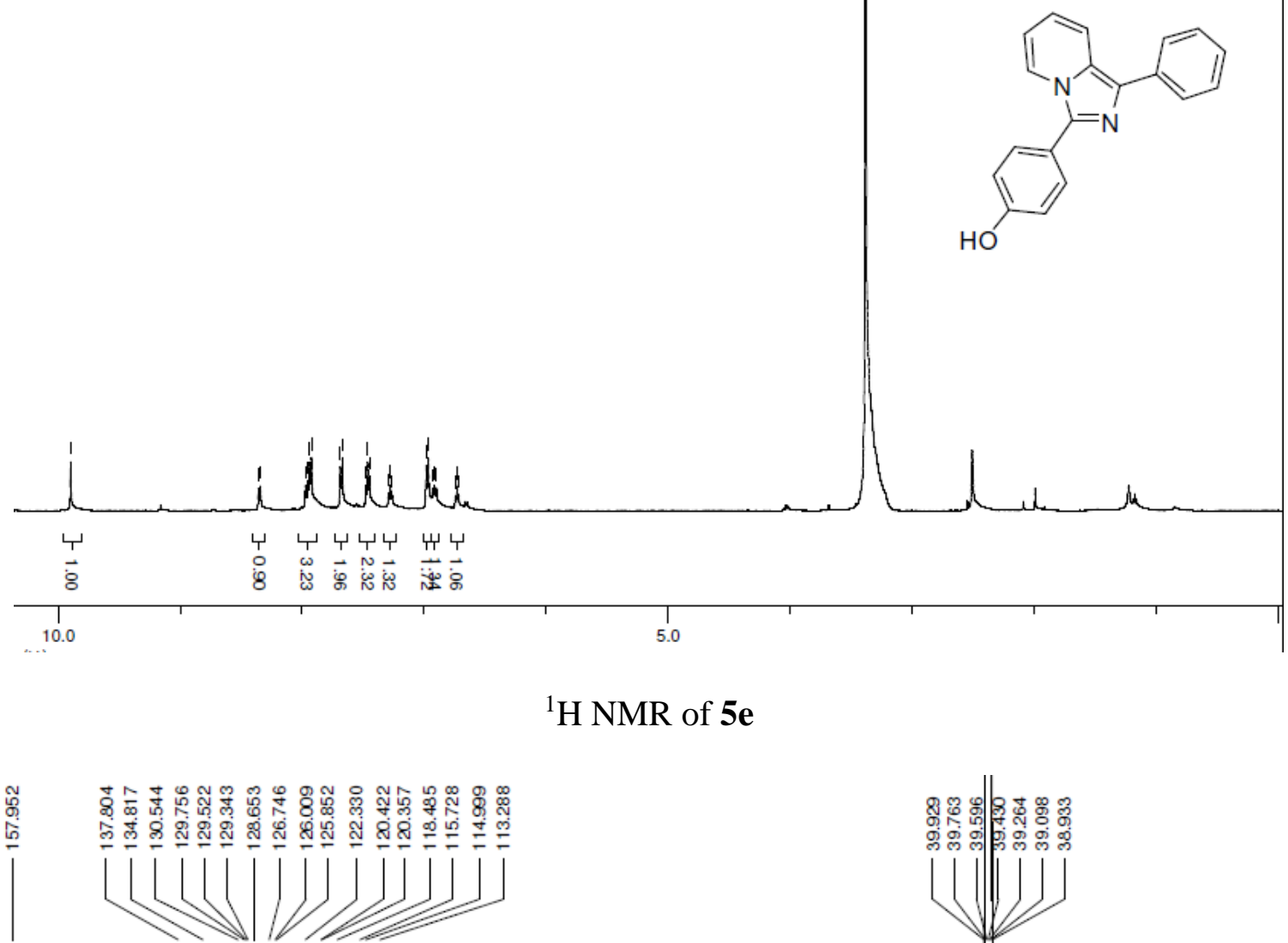

AKM-50

13C NMR in DMSO-d6, $500 \mathrm{MHz}$

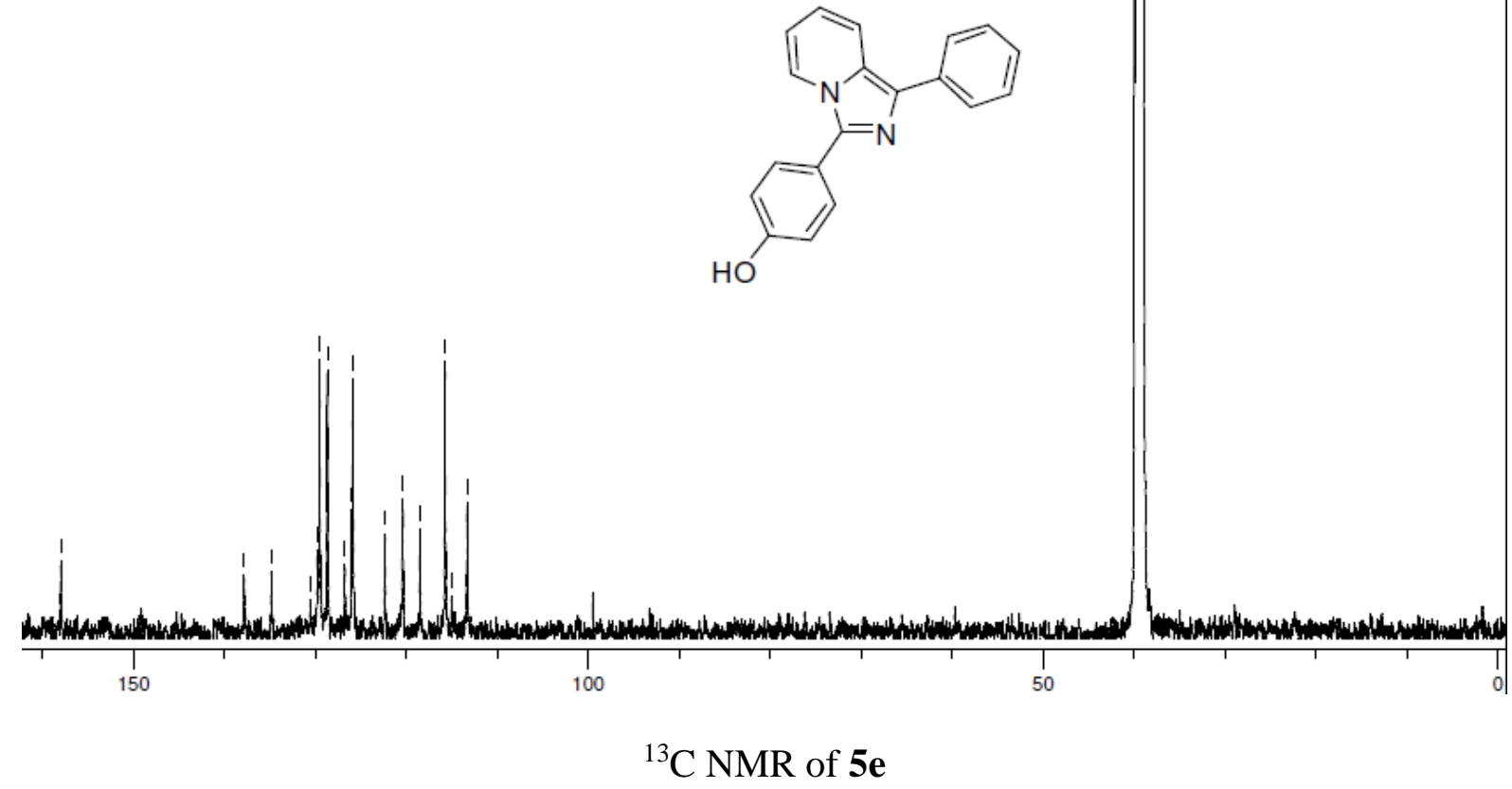




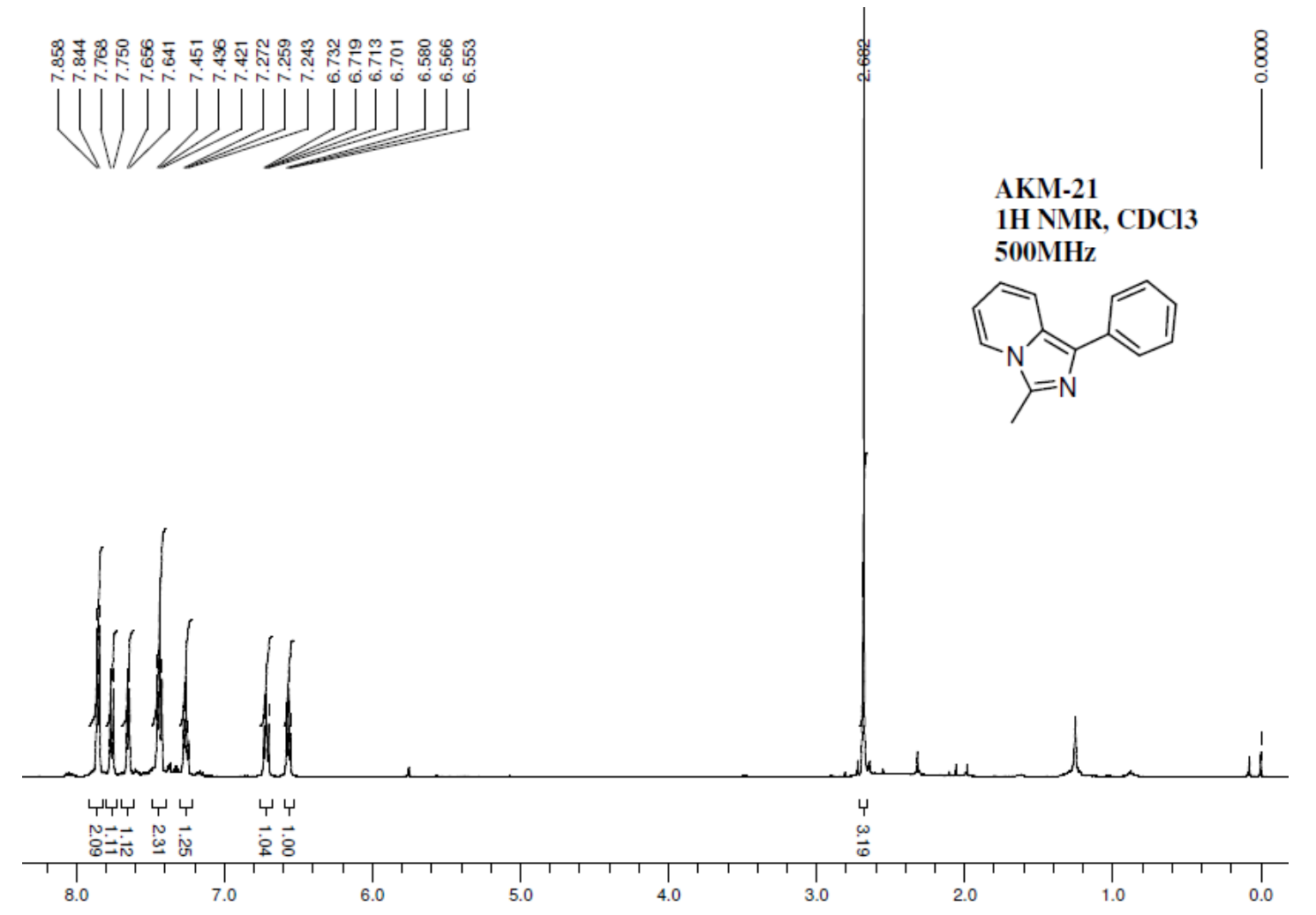

${ }^{1}$ H NMR of $\mathbf{5 f}$

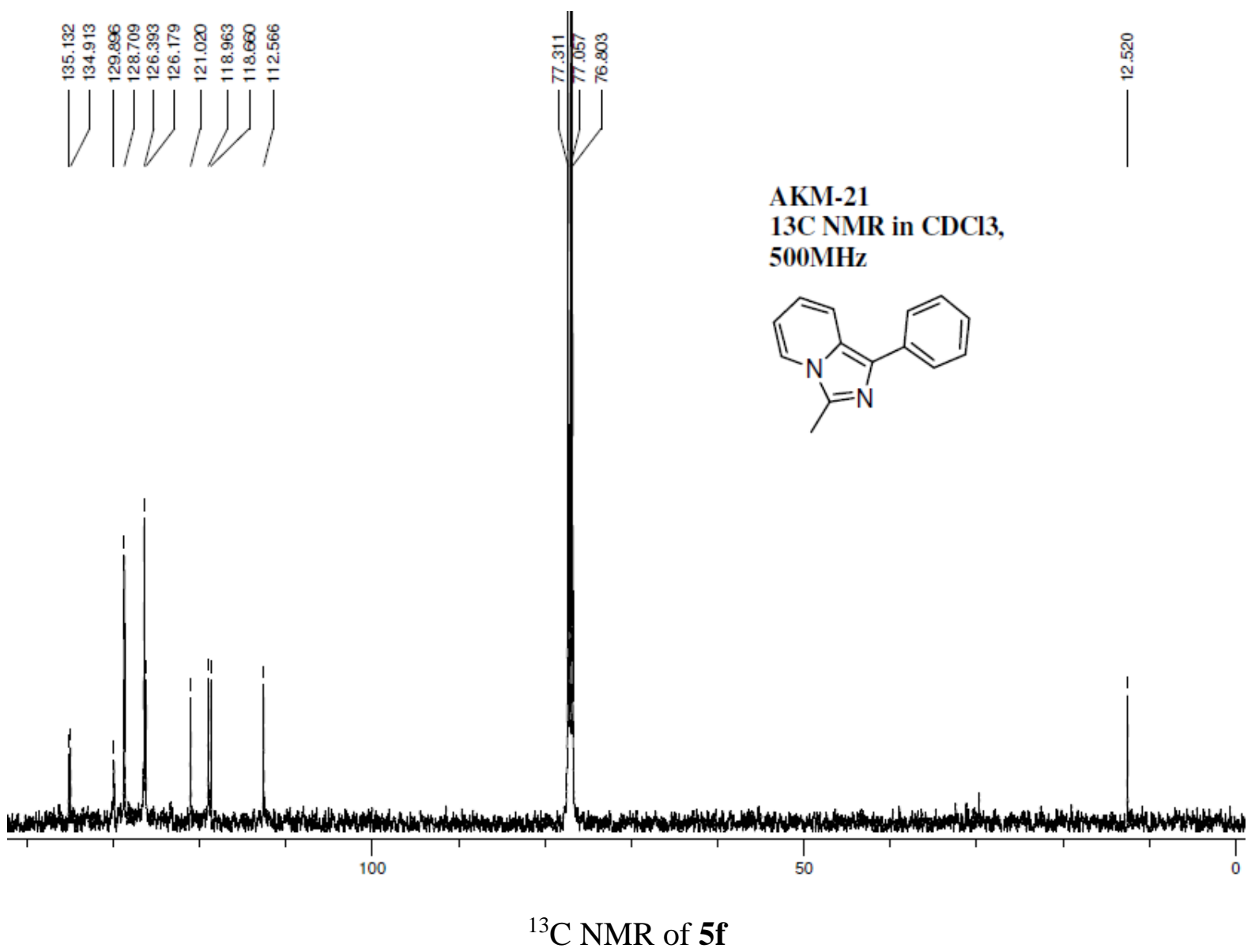


AKM-34

$1 \mathrm{H} \mathrm{NMR} \mathrm{in} \mathrm{CDCl} 3$,

$500 \mathrm{MHz}$<smiles>CC(C)Cc1nc(-c2ccccc2)c2ccccn12</smiles>
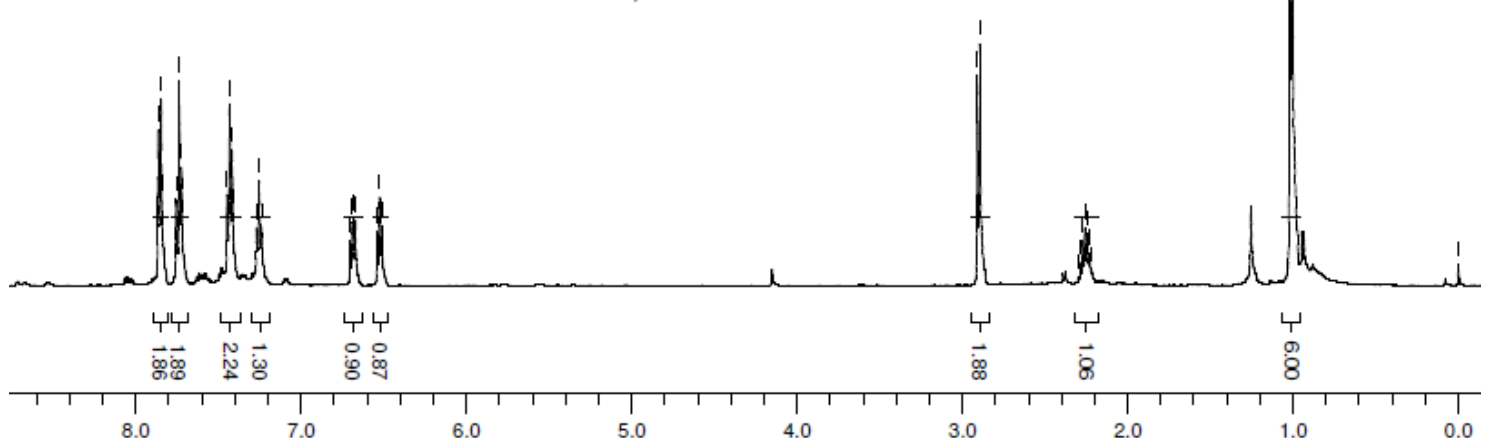

${ }^{1} \mathrm{H}$ NMR of $\mathbf{5 g}$
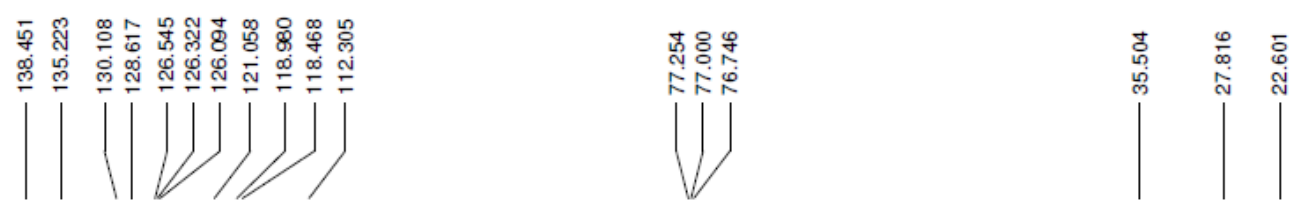

AKM-34

13C NMR in CDCl3,

$500 \mathrm{MHz}$
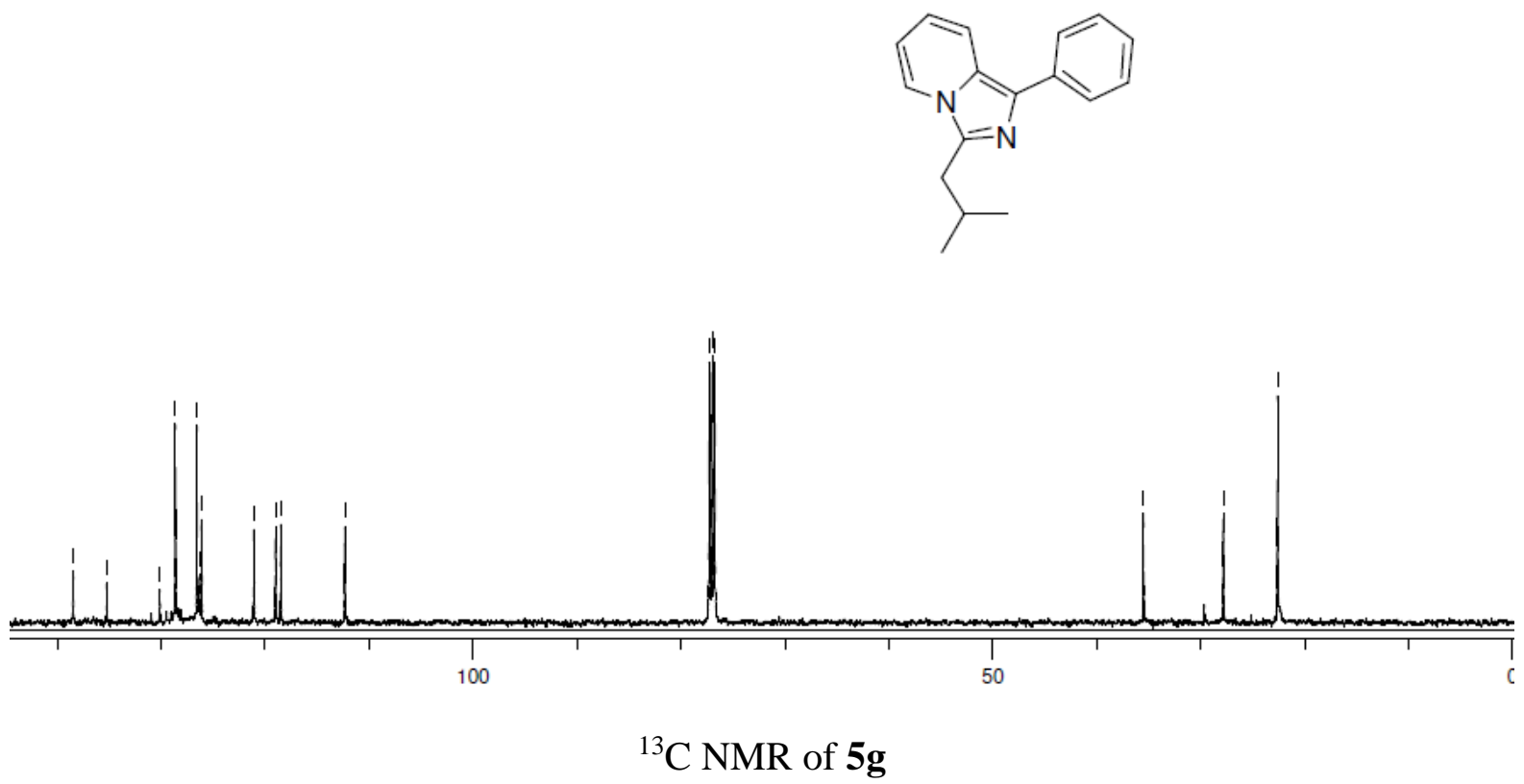